\title{
Incremental update of rough set approximation under the grade indiscernibility relation
}

\author{
Junfang Luo ${ }^{1}$, Yaya Liu ${ }^{1}$, Keyun Qin ${ }^{1 *}$ Heng Ding ${ }^{2}$ \\ ${ }^{1}$ College of Mathematics, Southwest Jiaotong University, Chengdu, Sichuan, 610031, China, \\ E-mail: junfangluo@163.com(Luo),1109731034@qq.com(Liu),keyunqin@263.net(Qin), \\ ${ }^{2}$ School of transportation and logistics, Southwest Jiaotong University, Chengdu, Sichuan, 610031, China, \\ E-mail:dh_swjtu@163.com(Ding).
}

Received 28 March 2016

Accepted 30 September 2016

\begin{abstract}
The incremental updating of lower and upper approximations under the variation of information systems is an important issue in rough set theory. Many incremental updating approaches with respect to different kinds of indiscernibility relations have been proposed. The grade indiscernibility relation is a fuzzification of classical Pawlak's indiscernibility relation which can characterize the similarity between objects more precisely. Based on fuzzy rough set model, this paper discusses the approaches for dynamically acquiring of the upper and lower approximations with respect to the grade indiscernibility relation when adding and removing an attribute or an object, and changing the attribute value of the object, respectively. Since the approaches are used in succession, they make the approximations can be updated correctly and effectively when any kind of possible change in the information system. Finally, extensive experiments on data sets from University of California, Irvine (UCI) show that the incremental methods effectively reduce the computing time in comparison with the traditional non-incremental method.
\end{abstract}

Keywords: Rough set, Fuzzy relation, The grade indiscernibility relation, Incremental learning, Approximation operators.

\section{Introduction}

Rough set theory, a mathematical tool for dealing with vagueness and uncertainty, was introduced by Pawlak in $1982^{1}$. It can be used in attribute value representation models to describe the dependencies among attributes, evaluate the significance of attributes and derive decision rules $2,3,4,5,6$. Rough setbased data analysis starts from a data table, also called an information system, which contains data about objects of interest that are characterized by a finite set of attributes. Objects with the same information are indiscernible and the indiscernibility re- lation generated in this way forms the mathematical basis for the theory of rough sets. By using the indiscernibility relation, a rough set is characterized by a pair of sets, called the lower and upper approximations. In recent years, classical rough sets have been extended to several general models, such as covering rough set model ${ }^{7}$, fuzzy rough set model $^{8}$, variable precision rough set model $^{9}$, generalized rough set mode ${ }^{10}$, probabilistic rough set mode ${ }^{11}$, etc.

With the rapid development of modern information technology, different types of data have increased dramatically. In many real-time cases, information systems may evolve over time, in other

\footnotetext{
* Corresponding author.
} 
words, some new information becomes available continuously while some information is no longer useful. One can retrain the system from scratch whenever adding or removing data(attributes or objects), which is known as a non-incremental approach $^{12}$. However, the non-incremental approach becomes very costly or even intractable as the number of data grows. Alternatively, one can also apply an incremental learning scheme ${ }^{12}$. The essence of incremental learning is to allow the learning process to take place in a continuous and progressive manner rather than a one-shot experience ${ }^{13}$. The research on updating knowledge incrementally has shown its importance in many areas, such as clinical decision making, intrusion detection, stock evaluation, and text categorization ${ }^{14}$. Some incremental learning methods with respect to rough set theory have been proposed ${ }^{14,15,16,17}$. Chan firstly put forward an incremental method for updating the approximations of a crisp concept based on the lower and upper boundary sets ${ }^{15}$. Li et al. presented an incremental method of updating decision rules when multi-attributes are deleted or added simultaneously under rough set based on the characteristic relation ${ }^{16}$. Zhang et al. investigated the approach for updating approximations under neighborhood rough sets ${ }^{17}$. Cheng proposed two incremental methods for the fast computing of the rough fuzzy approximations based on the boundary set and the cut sets of a fuzzy set, respectively ${ }^{14}$. These studies have significantly enriched the theory of rough set and guided a way for dynamic data mining, even big data mining.

The indiscernibility relation is a key notion of rough set theory, which partitions the object set of an information system into a collection of equivalence classes. Zhao proposed the notion of grade indiscernibility relation which is a fuzzy relation for information system to characterize the difference between the grades of discernibility ${ }^{18}$. Based on fuzzy rough set $\operatorname{model}^{8}$, Qin investigated rough approximation operators based on the grade indiscernibility relation ${ }^{19}$. For the sake of better applying of the grade indiscernibility relation, Luo extend it to incomplete information system ${ }^{20}$. The value tolerance relation based rough approximation opera- tors are investigated ${ }^{21}$. In this way, we defined the approximation operators based on the grade indiscernibility relation in the same manner ${ }^{20}$. Furthermore, the rule acquisition and attribute reduction are discussed, and the advantages of the grade of indiscernibility relation are also explained. In this paper, we discusses the approaches for incrementally acquiring approximations based on the grade indiscernibility relation when the information system changes. Due to these approaches are used in succession, they can effectively updated approximations when any possible changes in the information system occur. In order to show the succession of the approaches, examples in this paper are used as input from the output of the example before it. And it should be noted that the order of the information system changes can be arbitrary.

This paper is arranged as follows. In Section 2, we review some fundamental concepts of Pawlak rough sets and the grade indiscernibility relation. The remainders of the sections are focused on the approaches for incrementally updating approximations based on the grade indiscernibility relation when the information system varies with time. With changes of the attribute set, we discuss how to acquire approximation operators in Section 3. In Section 4, we investigate the methods for updating approximations when adding or removing an object in the universal set. The approaches for updating approximations when changing the attribute value of object is given in Section 5. In Section 6, we analyze the time complexity of algorithms presented in Section 3,4,5. In Section 7, the incremental methods are evaluated on data sets from UCI. Finally we conclude the work of this paper and preview the further work.

\section{Preliminaries}

In this section, for our further development, we briefly review some basic notions of Pawlak rough set $^{1}$ and the grade indiscernibility relation ${ }^{18}$. Meanwhile, the traditional non-incremental algorithm of calculating approximations is presented. 


\subsection{The grade indiscernibility relation}

Definition $1{ }^{1}$ An information system is a quadruple $S=(U, A, V, F)$, where

(1) $U=\left\{x_{1}, x_{2}, \cdots, x_{n}\right\}$ is a nonempty finite set of objects called the universe of discourse;

(2) A is a nonempty finite set of attributes;

(3) $V=\cup_{a \in A} V_{a}, V_{a}$ is the values domain of $a$;

(4) $f: U \times A \rightarrow V$ is an information function such that $f(x, a) \in V_{a}$, for any $x \in U, a \in A$.

Definition $2{ }^{1}$ Let $S=(U, A, V, F)$ be an information system, $B \subseteq A$, the indiscernibility relation ind $(B)$ induced by $B$ is defined as:

$\operatorname{ind}(B)=\{(x, y) \in U \times U ; \forall b \in B(f(x, b)=$ $f(y, b))\}$.

Clearly, $\operatorname{ind}(B)$ is an equivalence relation. If $(x, y) \in \operatorname{ind}(B)$, then $x$ and $y$ are indiscernible with respect to $B$. It is noticed that, if $x$ and $y$ are discernible with respect to $B$, i.e. $(x, y) \notin$ ind $(B)$, then there exists at least one attribute $b \in B$ such that $f(x, b) \neq f(y, b)$. Thus the grade of discernibility may be different for different pairs of objects. The difference has not been described in Pawlak's indiscernibility relation. To address this issue, Zhao proposed the grade indiscernibility relation for information system ${ }^{18}$, which is defined as follows.

Definition $3{ }^{18}$ Let $S=(U, A, V, F)$ be an information system, $B \subseteq A$, the grade indiscernibility relationship $G R_{B}$ on $B$ is a binary fuzzy relation on $U$, i.e. $G R_{B}: U \times U \rightarrow[0,1]$, and for any $x, y \in U$,

$G R_{B}(x, y)=\frac{1}{|B|}|\{b \in B ; f(x, b)=f(y, b)\}|$.

According to this definition, $G R_{B}(x, y)$ represents the proportion of undistinguishable attribute of $x$ and $y$ in $B$. Clearly, $(x, y) \in \operatorname{ind}(B)$ if and only if $G R_{B}(x, y)=1$. Thus, $G R_{B}$ is a kind of fuzzification of a indiscernibility relation $\operatorname{ind}(B)$. It is noticed that $G R_{B}$ is a reflexive and symmetric fuzzy relation, but not necessarily transitive, i.e., $G R_{B}(x, y) \wedge$ $G R_{B}(y, z) \leqslant G R_{B}(x, z)$ is not necessarily hold. From the point of fuzzy rough set model ${ }^{8}$, Qin presented approximation operators based on grade indiscernibility relation ${ }^{19}$.

Definition $4{ }^{19}$ Let $S=(U, A, V, F)$ be an information system, $B \subseteq A$. For any fuzzy subset $\mu \in F(U)$, the lower approximation $\underline{G R}_{B}(\mu)$ and the upper ap- proximations $\overline{G R}_{B}(\mu)$ of $\mu$ are defined as follows: $\forall x \in U$,

$$
\begin{aligned}
& \underline{G R}_{B}(\mu)(x)=\underset{y \in U}{\wedge}\left(\left(1-G R_{B}(x, y)\right) \vee \mu(y)\right) ; \\
& \overline{G R}_{B}(\mu)(x)=\underset{y \in U}{\vee}\left(G R_{B}(x, y) \wedge \mu(y)\right) .
\end{aligned}
$$

In this definition, if $X \in P(U)$ is a subset of $U$, then

$$
\begin{aligned}
& \underline{G R}_{B}(X)(x)=\underset{y \in U-X}{\wedge}\left(1-G R_{B}(x, y)\right), \\
& \overline{G R}_{B}(X)(x)=\vee_{y \in X}^{\vee} G R_{B}(x, y) .
\end{aligned}
$$

Clearly, by the reflexivity of $G R_{B}(x, y)$, we have $\underline{G R}_{B}(X) \subseteq X \subseteq \overline{G R}_{B}(X)$ for any $X \subseteq U$.

\subsection{The non-incremental algorithm of computing approximations}

In order to get the approximations based on the grade indiscernibility relation, the non-incremental method will firstly build the relation matrix and then get the approximations from the matrix. The calculation process of the traditional way can be represented as the following Steps in Algorithm 2.1.

Algorithm 2.1 (The traditional non-incremental algorithm of computing approximations)

Step 1: Input $S=(U, A, V, f), X, B$.

Step 2: Build the relation matrix $M_{n \times m}$, where $n=|X|, m=|U-X|$.

$M_{n \times m}=G R_{B}\left(x_{i}, x_{j}\right)=\frac{1}{|B|}\left|\left\{b \in B \mid, f\left(x_{i}, b\right)=f\left(x_{j}, b\right)\right\}\right|$, $x_{i} \in X, x_{j} \in U-X$.

Step 3: Calculate the lower approximations $\underline{G R}_{B}(X)\left(x_{i}\right)=\underset{y \in U-X}{\wedge}\left(1-G R_{B}\left(x_{i}, y\right)\right)=1-$ $\underset{y \in U-X}{\vee} G R_{B}\left(x_{i}, y\right)$ and the set $Y_{x_{i}}^{\wedge}=\{y \in U-$ $\left.X ; \underset{y \in U-X}{\vee} G R_{B}\left(x_{i}, y\right)\right\}, x_{i} \in X$

Step 4: Calculate the upper approximations $\overline{G R}_{B}(X)\left(x_{j}\right)=\underset{y \in X}{\vee} G R_{B}\left(x_{j}, y\right)$ and the set $Y_{x_{j}}^{\vee}=\{y \in$ $\left.X ; \underset{y \in X}{\vee} G R_{B}\left(x_{j}, y\right)\right\}, x_{j} \in U-X$.

Step 5: Output the $M_{n \times m} ; \underline{G R}_{B}(X)\left(x_{i}\right)$ and $Y_{x_{i}}^{\wedge}$, $x_{i} \in X ; \overline{G R}_{B}(X)\left(x_{j}\right.$ and $Y_{x_{j}}^{\vee}, x_{j} \in U-X ; \underline{G R}_{B}(X)\left(x_{i}\right)=$ $0, x_{i} \in U-X ; \overline{G R}_{B}(X)\left(x_{j}=1, x_{j} \in X\right.$.

It is easy to see that Algorithm 2.1 has a time complexity of $O(|X||U-X||B|)$, which is mainly decided by the time cost of building the relation matrix in Step 2. The following Example 1 shows the 
progress of using the Algorithm 2.1 to get the approximations based on the grade indiscernibility relation.

Example 1 Let $U=\left\{x_{1}, x_{2}, x_{3}, x_{4}, x_{5}, x_{6}\right\}$ be the universal set, $A=\left\{b_{1}, b_{2}, b_{3}, b_{4}, b_{5}\right\}$ be the conditional attribute set, $B=\left\{b_{1}, b_{2}, b_{3}, b_{4}\right\}, X=\left\{x_{2}, x_{3}, x_{6}\right\}$ be the decision set. The related information system is given in Table 1.

\begin{tabular}{ccccccc}
\multicolumn{8}{c}{ Table 1.} \\
\hline & $b_{1}$ & $b_{2}$ & $b_{3}$ & $b_{4}$ & $b_{5}$ & $d$ \\
\hline$x_{1}$ & 2 & 2 & 1 & 1 & 3 & $U-X$ \\
$x_{2}$ & 2 & 2 & 1 & 2 & 2 & $X$ \\
$x_{3}$ & 2 & 2 & 2 & 1 & 3 & $X$ \\
$x_{4}$ & 1 & 1 & 1 & 2 & 2 & $U-X$ \\
$x_{5}$ & 1 & 2 & 2 & 2 & 3 & $X$ \\
$x_{6}$ & 1 & 1 & 2 & 2 & 2 & $U-X$ \\
\hline
\end{tabular}

From the Step 2 of Algorithm 2.1 we have a relation matrix $M_{3 \times 3}$, where $G R_{B}(x, y), x_{i} \in X, x_{j} \in U-X$.

$$
\begin{array}{r}
x_{1} \\
M_{3 \times 3}=x_{2} \\
x_{3} \\
x_{6}
\end{array}\left(\begin{array}{ccc}
3 / 4 & 2 / 4 & 2 / 4 \\
3 / 4 & 0 & 2 / 4 \\
0 & 3 / 4 & 3 / 4
\end{array}\right)
$$

The first column is the object that $x_{i} \in X$. The first row is the object that $x_{j} \in U-X$.

From the step 3 of algorithm 2.1 we have the lower approximations:

$$
\begin{aligned}
& \underline{G R}_{B}(X)\left(x_{2}\right)=\hat{y}_{y \in U-X}\left(1-G R_{B}\left(x_{2}, y\right)=1-\right. \\
& \underset{y \in U-X}{\vee} G R_{B}\left(x_{2}, y\right)=1-\frac{3}{4}=\frac{1}{4}, \quad \text { and } \quad Y_{x_{2}}^{\wedge}= \\
& \left\{y ; \underset{y \in U-X}{\vee} G R_{B}\left(x_{2}, y\right)\right\}=\left\{x_{1}\right\} \text {. } \\
& \underline{G R}_{B}(X)\left(x_{3}\right)=\wedge_{y \in U-X}\left(1-G R_{B}\left(x_{3}, y\right)=1-\right. \\
& \underset{y \in U-X}{\vee} G R_{B}\left(x_{3}, y\right)=1-\frac{3}{4}=\frac{1}{4} \text {, and } Y_{x_{3}}^{\wedge}= \\
& \left\{y ; \underset{y \in U-X}{\vee} G R_{B}\left(x_{3}, y\right)\right\}=\left\{x_{1}\right\} \text {. } \\
& \underline{G R}_{B}(X)\left(x_{6}\right)=\wedge_{y \in U-X}\left(1-G R_{B}\left(x_{6}, y\right)=1-\right. \\
& \underset{y \in U-X}{\vee} G R_{B}\left(x_{6}, y\right)=1-\frac{3}{4}=\frac{1}{4}, \quad \text { and } \quad Y_{x_{6}}^{\wedge}= \\
& \left\{y ; \underset{y \in U-X}{\vee} G R_{B}\left(x_{6}, y\right)\right\}=\left\{x_{4}, x_{5}\right\} \text {. } \\
& \underline{G R}_{B}(X)\left(x_{i}\right)=0, x_{i} \in\left\{x_{1}, x_{4}, x_{5}\right\} .
\end{aligned}
$$

From the step 4 of algorithm 2.1 we have the upper approximations:

$$
\overline{G R}_{B}(X)\left(x_{1}\right)=\underset{y \in X}{\vee} G R_{B}\left(x_{1}, y\right)=\frac{3}{4} \text {, and } Y_{x_{1}}^{\vee}=\{y \in
$$

$\left.X ; \underset{y \in X}{\vee} G R_{B}\left(x_{1}, y\right)\right\}=\left\{x_{2}, x_{3}\right\}$.

$$
\overline{G R}_{B}(X)\left(x_{4}\right)=\underset{y \in X}{\vee} G R_{B}\left(x_{4}, y\right)=\frac{3}{4} \text {, and } Y_{x_{4}}^{\vee}=\{y \in
$$

$\left.X ; \underset{y \in X}{\vee} G R_{B}\left(x_{4}, y\right)\right\}=\left\{x_{6}\right\}$.

$$
\overline{G R}_{B}(X)\left(x_{5}\right)=\underset{y \in X}{\vee} G R_{B}\left(x_{5}, y\right)=\frac{3}{4} \text {, and } Y_{x_{5}}^{\vee}=\{y \in
$$

$\left.X ; \underset{y \in X}{\vee} G R_{B}\left(x_{5}, y\right)\right\}=\left\{x_{6}\right\}$.

$$
\overline{G R}_{B}(X)\left(x_{j}\right)=1, x_{j} \in\left\{x_{2}, x_{3}, x_{6}\right\} .
$$

\section{Incrementally updating approximations while adding or removing an attribute}

The traditional non-incrementally update method is based on static information system, which has huge time complexity as the number of data grows. Incremental update method can improve the efficiency by using the existing approximation knowledge ${ }^{18}$.

\subsection{Incrementally updating approximations when adding an attribute}

Proposition 1 Let $S=(U, A, V, F)$ be an information system, $B \subseteq A, b \in A$ and $b \notin B$. For any $x, y \in U$,

$$
\begin{aligned}
& G_{B \cup\{b\}}(x, y)=\frac{|B| \cdot G R_{B}(x, y)+G R_{\{b\}}(x, y)}{|B|+1} \\
& = \begin{cases}\frac{|B| \cdot G R_{B}(x, y)+1}{|B|+1}, & f(x, b)=f(y, b) ; \\
\frac{|B| \cdot G R_{B}(x, y)}{|B|+1}, & f(x, b) \neq f(y, b) .\end{cases}
\end{aligned}
$$

Proof. This proof is straightforward.

Proposition 2 Let $S=(U, A, V, F)$ be an information system, $B \subseteq A, b \in A, b \notin B, X \subseteq U$. The lower and upper approximations of $X$ by adding $b$ to $B$ can be updated respectively as follows.

Lower approximation: If $x_{i} \in U-X$, then $\underline{G R}_{B \cup\{b\}}(X)\left(x_{i}\right)=\underline{G R}_{B}(X)\left(x_{i}\right)=0$; If $x_{i} \in X$, then $\underline{G R}_{B \cup\{b\}}(X)\left(x_{i}\right)$

$$
= \begin{cases}\frac{|B| G R_{B}(X)\left(x_{i}\right)}{|B|+1}, & \exists y \in Y_{x_{i}}^{\wedge}\left(f\left(x_{i}, b\right)=f(y, b)\right) ; \\ \frac{1+|B| \underline{G} R_{B}(X)\left(x_{i}\right)}{|B|+1}, & \forall y\left(y \in Y_{x_{i}}^{\wedge} \rightarrow f\left(x_{i}, b\right) \neq f(y, b)\right) .\end{cases}
$$

Where $Y_{x_{i}}^{\wedge}=\left\{y \in U-X ; \underline{G R}_{B}(X)\left(x_{i}\right)=1-\right.$ $\left.G R_{B}\left(x_{i}, y\right)\right\}=\left\{y ; \underset{y \in U-X}{\vee} G R_{B}\left(x_{i}, y\right)\right\}$.

Upper approximation: If $x_{j} \in X$, then $\overline{G R}_{B \cup\{b\}}(X)\left(x_{j}\right)=\overline{G R}_{B}(X)\left(x_{j}\right)=1$; If $x_{j} \in U-X$, then $\overline{G R}_{B \cup\{b\}}(X)\left(x_{j}\right)$ 


$$
= \begin{cases}\frac{|B| \overline{G R}_{B}(X)\left(x_{j}\right)+1}{|B|+1}, & \exists y \in Y_{x_{j}}^{\vee}\left(f\left(x_{j}, b\right)=f(y, b)\right) ; \\ \frac{|B| G R_{B}(X)\left(x_{j}\right)}{|B|+1}, & \forall y\left(y \in Y_{x_{j}}^{\vee} \rightarrow f\left(x_{j}, b\right) \neq f(y, b)\right) .\end{cases}
$$

Proof. Lower approximation: For any $x_{i} \in U-X$, It is obviously that $\underline{G R}_{B \cup\{b\}}(X)\left(x_{i}\right)=\underline{G R}_{B}(X)\left(x_{i}\right)=0$.

For any $x_{i} \in X$, if $\exists y \in Y_{x_{i}}^{\wedge}$ such that $f\left(x_{i}, b\right)=$ $f(y, b)$, then $\underline{G R}_{B \cup\{b\}}(X)\left(x_{i}\right)=1-G R_{B \cup\{b\}}\left(x_{i}, y\right)=$ $1-\frac{|B| G R_{B}\left(x_{i}, y\right)+1}{|B|+1}=1-\frac{|B|\left(1-G R_{B}(X)\left(x_{i}\right)\right)+1}{|B|+1}=\frac{|B| G R_{B}(X)\left(x_{i}\right)}{|B|+1}$.

If $f\left(x_{i}, b\right) \neq f(y, b)$ for any $y \in Y_{x_{i}}^{\wedge}$, then $\underline{G R}_{B \cup\{b\}}(X)\left(x_{i}\right)=\bigwedge_{y \in U-X}\left(1-G R_{B \cup\{b\}}\left(x_{i}, y\right)\right)=1-$ $\left(G R_{B \cup\{b\}}\left(x_{i}, y_{0}\right) \vee\left(\underset{y \in U-X-Y_{x_{i}}^{\wedge}, f\left(x_{i}, b\right)=f(y, b)}{\vee} G R_{B \cup\{b\}}\left(x_{i}, y\right)\right)\right)=$ $1-\left(\frac{|B| G R_{B}\left(x_{i}, y_{0}\right)}{|B|+1} \vee\left({ }_{y \in U-X-Y_{x_{i}}^{\wedge}, f\left(x_{i}, b\right)=f(y, b)} \frac{|B| G R_{B}\left(x_{i}, y\right)+1}{|B|+1}\right)\right.$, where $y_{0} \in Y_{x_{i}}^{\wedge}$. For any $y \in U-X-Y_{x_{i}}^{\wedge}$, we have $G R_{B}\left(x_{i}, y_{0}\right)>G R_{B}\left(x_{i}, y\right)$, that is $\frac{|B| G R_{B}\left(x_{i}, y_{0}\right)}{|B|+1} \geqslant$ $\frac{|B| G R_{B}\left(x_{i}, y\right)+1}{|B|+1}$. Therefore, $\quad \underline{G R}_{B \cup\{b\}}(X)\left(x_{i}\right)=1$ $\frac{|B| G R_{B}\left(x_{i}, y_{0}\right)}{|B|+1}=\frac{|B| G R_{B}(X)\left(x_{i}\right)+1}{|B|+1}$.

Upper approximation: For any $x_{j} \in X$, it is obviously that $\overline{G R}_{B \cup\{b\}}(X)\left(x_{j}\right)=\overline{G R}_{B}(X)\left(x_{j}\right)=1$.

For any $x_{j} \in U-X$, if $\exists y \in Y_{x_{j}}^{\vee}$, such that $f\left(x_{j}, b\right)=f(y, b)$, then $\overline{G R}_{B \cup\{b\}}(X)\left(x_{j}\right)=$ $G R_{B \cup\{b\}}\left(x_{j}, y\right)=\frac{|B| G R_{B}\left(x_{j}, y\right)+1}{|B|+1}=\frac{|B| G R_{B}(X)\left(x_{j}\right)+1}{|B|+1}$.

If $f\left(x_{j}, b\right) \neq f(y, b)$ for any $y \in Y_{x_{j}}^{\vee}$, then $\quad \overline{G R}_{B \cup\{b\}}(X)\left(x_{j}\right)=\underset{y \in X}{\vee} G R_{B \cup\{b\}}\left(x_{j}, y\right)=$ $G R_{B \cup\{b\}}\left(x_{j}, y_{0}\right) \vee\left(\underset{y \in X-Y_{x_{j}}, f\left(x_{j}, b\right)=f(y, b)}{\vee} G R_{B \cup\{b\}}\left(x_{j}, y\right)\right)=$ $\frac{|B| G R_{B}\left(x_{j}, y_{0}\right)}{|B|+1} \quad \vee \quad\left(\underset{y \in X-Y_{x_{j}}^{\vee}, f\left(x_{j}, b\right)=f(y, b)}{\vee} \frac{|B| G R_{B}\left(x_{j}, y\right)+1}{|B|+1}\right)$, where $y_{0} \in Y_{x_{j}}^{\vee}$. For any $y \in X-Y_{x_{j}}^{\vee}$, we have $G R_{B}\left(x_{j}, y_{0}\right)>G R_{B}\left(x_{j}, y\right)$, that is $\frac{|B| G R_{B}\left(x_{j}, y_{0}\right)}{|B|+1} \geqslant$ $\frac{|B| G R_{B}\left(x_{j}, y\right)+1}{|B|+1}$. Therefore, $\overline{G R}_{B \cup\{b\}}(X)\left(x_{j}\right)=$ $\frac{|B| G R_{B}\left(x_{j}, y_{0}\right)}{|B|+1}=\frac{|B| G R_{B}(X)\left(x_{j}\right)}{|B|+1}$.

Particularly, if $Y_{x_{i}}^{\wedge}=\left\{y_{k}\right\}$ and $Y_{x_{j}}^{\vee}=\left\{y_{l}\right\}$ are subsets of the universe with single element, the lower and upper approximations of $X$ by adding $b$ to $B$ can be updated respectively as follows.

Lower approximation: If $x_{i} \in U-X$, then $\underline{G R}_{B \cup\{b\}}(X)\left(x_{i}\right)=\underline{G R}_{B}(X)\left(x_{i}\right)=0$; If $x_{i} \in X$, then $\underline{G R}_{B \cup\{b\}}(X)\left(x_{i}\right)= \begin{cases}\frac{|B| G R_{B}(X)\left(x_{i}\right)}{|B|+1}, & f\left(x_{i}, b\right)=f\left(y_{k}, b\right) ; \\ \frac{|B| G R_{B}(X)\left(x_{i}\right)+1}{|B|+1}, & f\left(x_{i}, b\right) \neq f\left(y_{k}, b\right) .\end{cases}$
Upper approximation: If $x_{j} \in X$, then $\overline{G R}_{B \cup\{b\}}(X)\left(x_{j}\right)=\overline{G R}_{B}(X)\left(x_{j}\right)=1$; If $x_{j} \in U-X$, then

$\overline{G R}_{B \cup\{b\}}(X)\left(x_{j}\right)= \begin{cases}\frac{|B| \overline{G R}_{B}(X)\left(x_{j}\right)+1}{|B|+1}, & f\left(x_{j}, b\right)=f\left(y_{l}, b\right) ; \\ \frac{|B| G R_{B}(X)\left(x_{j}\right)}{|B|+1}, & f\left(x_{j}, b\right) \neq f\left(y_{l}, b\right) .\end{cases}$

Since the incremental update approaches need to use the set of $Y_{x_{i}}^{\wedge}$ and $Y_{x_{j}}^{\vee}$, in order to maintain the continuity of the approaches, $Y_{x_{i}}^{\wedge}$ and $Y_{x_{j}}^{\vee}$ also need to update.

Proposition 3 The set of $Y_{x_{i}}^{\wedge}$ and $Y_{x_{i}}^{\vee}$ by adding $b$ to $B$ can be updated respectively as follows.

$$
\begin{aligned}
& Y_{x_{i}}^{\wedge *}=\left\{\begin{array}{l}
\left\{y \in Y_{x_{i}}^{\wedge} ; f\left(x_{i}, b\right)=f(y, b)\right\}, \exists y \in Y_{x_{i}}^{\wedge} \\
\left(f\left(x_{i}, b\right)=f(y, b)\right) ; \\
\left\{y ; \underset{y \in U-X}{\vee} G R_{B \cup\{b\}}\left(x_{i}, y\right)\right\}, \forall y\left(y \in Y_{x_{i}}^{\wedge}\right. \\
\left.\rightarrow f\left(x_{i}, b\right) \neq f(y, b)\right),
\end{array}\right. \\
& Y_{x_{j}}^{\vee *}=\left\{\begin{array}{l}
\left\{y \in Y_{x_{j}}^{\vee} ; f\left(x_{j}, b\right)=f(y, b)\right\}, \exists y \in Y_{x_{j}}^{\vee} \\
\left(f\left(x_{j}, b\right)=f(y, b)\right) ; \\
\left\{y ; \underset{y \in X}{\vee} G R_{B \cup\{b\}}\left(x_{j}, y\right)\right\}, \forall y\left(y \in Y_{x_{j}}^{\vee} \rightarrow\right. \\
\left.f\left(x_{j}, b\right) \neq f(y, b)\right) .
\end{array}\right.
\end{aligned}
$$

Algorithm 3.1 (Incremental algorithm for updating approximations when adding an attribute $b$ )

Step 1: Input the relation matrix $M_{n \times m}$; $\underline{G R}_{B}(X)\left(x_{i}\right), Y_{x_{i}}^{\wedge}, x_{i} \in X ; \overline{G R}_{B}(X)\left(x_{j}, Y_{x_{j}}^{\vee}, x_{j} \in U-X ;\right.$ the increasing attribute $b$.

Step 2: We get a new relation matrix $M_{n \times m}^{*}=$ $G R_{B \cup\{b\}}\left(x_{i}, x_{j}\right)= \begin{cases}\frac{|B| \cdot G R_{B}\left(x_{i}, x_{j}\right)+1}{|B|+1}, & f\left(x_{i}, b\right)=f\left(x_{j}, b\right) ; \\ \frac{|B| \cdot G R_{B}\left(x_{i}, x_{j}\right)}{|B|+1}, & f\left(x_{i}, b\right) \neq f\left(x_{j}, b\right) .\end{cases}$ $x_{i} \in X, x_{j} \in U-X$. // According to Proposition 1.

Step 3: Calculate the lower approximations $x_{i} \in$ $X$.

If $\exists y \in Y_{x_{i}}^{\wedge}$, such that $f\left(x_{i}, b\right)=f(y, b)$, then $\underline{G R}_{B \cup\{b\}}(X)\left(x_{i}\right)=\frac{|B| G R_{B}(X)\left(x_{i}\right)}{|B|+1}$ and $Y_{x_{i}}^{\wedge *}=\{y \in$ $\left.Y_{x_{i}}^{\wedge} ; f\left(x_{i}, b\right)=f(y, b)\right\}$.

Else $\quad \underline{G R}_{B \cup\{b\}}(X)\left(x_{i}\right)=\frac{1+|B| G R_{B}(X)\left(x_{i}\right)}{|B|+1}$ and $Y_{x_{i}}^{\wedge *}=\left\{y ; \underset{y \in U-X}{\vee} G R_{B \cup\{b\}}\left(x_{i}, y\right)\right\} . \quad / /$ According to Proposition 2 and 3.

Step 4: Calculate the upper approximations $x_{j} \in$ $U-X$ 
If $\exists y \in Y_{x_{j}}^{\vee}$, such that $f\left(x_{j}, b\right)=f(y, b)$ then $\overline{G R}_{B \cup\{b\}}(X)\left(x_{j}\right)=\frac{|B| \overline{G R}_{B}(X)\left(x_{j}\right)+1}{|B|+1}$ and $Y_{x_{j}}^{\vee *}=\{y \in$ $\left.Y_{x_{j}}^{\vee} ; f\left(x_{j}, b\right)=f(y, b)\right\}$.

Else $\quad \overline{G R}_{B \cup\{b\}}(X)\left(x_{j}\right)=\frac{|B| \overline{G R}_{B}(X)\left(x_{j}\right)}{|B|+1} \quad$ and $Y_{x_{j}}^{\vee^{*}}=\left\{y ; \underset{y \in X}{\vee} G R_{B \cup\{b\}}\left(x_{j}, y\right)\right\} . / /$ According to Proposition 2 and 3 .

Step 5: Output the relation matrix $M_{n \times m}^{*}$; $\underline{G R}_{B \cup\{b\}}(X)\left(x_{i}\right), Y_{x_{i}}^{\wedge *}, x_{i} \in X ; \overline{G R}_{B \cup\{b\}}(X)\left(x_{j}\right), Y_{x_{j} *}^{\vee *}$, $\bar{x}_{j} \in U-X ; \quad \underline{G R}_{B \cup\{b\}}(X)\left(x_{i}\right)=0, x_{i} \in U-X ;$ $\overline{G R}_{B \cup\{b\}}(X)\left(x_{j}\right)=1, x_{j} \in X$.

The time complexity of Algorithm 3.1 is $O(|X||U-X|)$, which is mainly decided by the time cost of building the relation matrix in Step 2. In the following Example 2, We use the results from Example 1 to demonstrate how algorithm 3.1 update the approximations when adding an attribute.

Example 2 We consider the information system given in Table 1. Let $U=\left\{x_{1}, x_{2}, x_{3}, x_{4}, x_{5}, x_{6}\right\}$ be the universal set, $B=\left\{b_{1}, b_{2}, b_{3}, b_{4}\right\}$ be the conditional attribute set, $X=\left\{x_{2}, x_{3}, x_{6}\right\}$ be the decision set. Adding an attribute $b_{5}$ to $B$.

Using the result of Example 1.

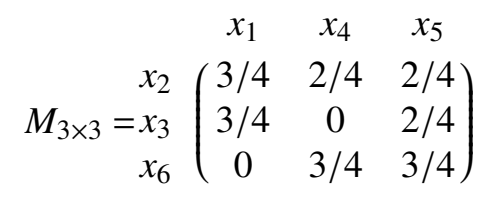

$\underline{G R}_{B}(X)\left(x_{2}\right)=\frac{1}{4}, Y_{x_{2}}^{\wedge}=\left\{x_{1}\right\} ; \underline{G R}_{B}(X)\left(x_{3}\right)=\frac{1}{4}, Y_{x_{3}}^{\wedge}=$ $\left\{x_{1}\right\} ; G R_{B}(X)\left(x_{6}\right)=\frac{1}{4}, Y_{x_{6}}^{\wedge}=\left\{x_{4}, x_{5}\right\} ; \underline{G R}_{B}(X)$

$\left(x_{i}\right)=0, x_{i} \in\left\{x_{1}, x_{4}, x_{5}\right\}$.

$\overline{G R}_{B}(X)\left(x_{1}\right)=\frac{3}{4}, Y_{x_{1}}^{\vee}=\left\{x_{2}, x_{3}\right\} ; \overline{G R}_{B}(X)\left(x_{4}\right)=$ $\frac{3}{4}, Y_{x_{4}}^{\vee}=\left\{x_{6}\right\} ; \overline{G R}_{B}(X)\left(x_{5}\right)=\frac{3}{4}, Y_{x_{5}}^{\vee}=\left\{x_{6}\right\} ; \overline{G R}_{B}(X)\left(x_{j}\right)$ $=1, x_{j} \in\left\{x_{2}, x_{3}, x_{6}\right\}$.

From the Step 2 of Algorithm 3.1 we have a new relation matrix $M_{3 \times 3}^{*}$ :

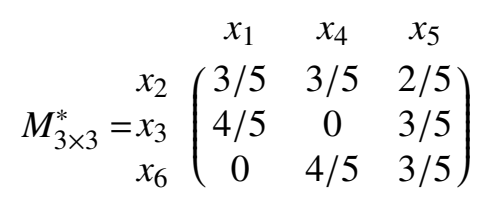

The lower and upper approximations of $X=$ $\left\{x_{2}, x_{3}, x_{6}\right\}$ by adding $b_{5}$ to $B$ are updated as follows.
From the step 3 of algorithm 3.1 we have the lower approximations:

$\underline{G R}_{B \cup\left\{b_{5}\right\}}(X)\left(x_{2}\right)$ : For any $y \in Y_{x_{2}}^{\wedge}, f\left(x_{2}, b_{5}\right) \neq$ $f\left(y, b_{5}\right)$. So we have $\underline{G R}_{B \cup\left\{b_{5}\right\}}(X)\left(x_{2}\right)=\frac{1+1}{4+1}=\frac{2}{5}$ and $Y_{x_{2}}^{\wedge *}=\left\{y ; \underset{y \in U-X}{\vee} G R_{B \cup\left\{b_{5}\right\}}\left(x_{2}, y\right)\right\}=\left\{x_{1}, x_{4}\right\}$.

$\underline{G R}_{B \cup\left\{b_{5}\right\}}(X)\left(x_{3}\right): \exists x_{1} \in Y_{x_{3}}^{\wedge}$, such that $f\left(x_{3}, b_{5}\right)=$ $f\left(x_{1}, b_{5}\right)$, then $\underline{G R}_{B \cup\left\{b_{5}\right\}}(X)\left(x_{3}\right)=\frac{1}{4+1}=\frac{1}{5}$ and $Y_{x_{3}}^{\wedge *}=$ $\left\{y \in Y_{x_{3}}^{\wedge} ; f\left(x, b_{5}\right)=f\left(y, b_{5}\right)\right\}=\left\{x_{1}\right\}$.

$\underline{G R}_{B \cup\left\{b_{5}\right\}}(X)\left(x_{6}\right): \exists x_{4} \in Y_{x_{6}}^{\wedge}$, such that $f\left(x_{6}, b_{5}\right)=$ $f\left(x_{4}, b_{5}\right)$, then $\underline{G R}_{B \cup\left\{b_{5}\right\}}(X)\left(x_{6}\right)=\frac{1}{4+1}=\frac{1}{5}$ and $Y_{x_{6}}^{\wedge *}=$ $\left\{y \in Y_{x_{6}}^{\wedge} ; f\left(x, b_{5}\right)=f\left(y, b_{5}\right)\right\}=\left\{x_{4}\right\}$.

$\underline{G R}_{B \cup\left\{b_{5}\right\}}(X)\left(x_{i}\right)=0, x_{i} \in\left\{x_{1}, x_{4}, x_{5}\right\}$.

From the step 4 of algorithm 3.1 we have the upper approximations:

$\overline{G R}_{B \cup\left\{b_{5}\right\}}(X)\left(x_{1}\right): \exists x_{2} \in Y_{x_{1}}^{\vee}$, such that $f\left(x_{1}, b_{5}\right)=$ $f\left(x_{2}, b_{5}\right)$, then $\overline{G R}_{B \cup\left\{b_{5}\right\}}(X)\left(x_{1}\right)=\frac{3+1}{4+1}=\frac{4}{5}$ and $Y_{x_{1}}^{\vee *}=$ $\left\{y \in Y_{x_{1}}^{\vee} ; f\left(x_{1}, b_{5}\right)=f\left(y, b_{5}\right)\right\}=\left\{x_{3}\right\}$.

$\overline{G R}_{B \cup\left\{b_{5}\right\}}(X)\left(x_{4}\right): \exists x_{6} \in Y_{x_{4}}^{\vee}$, such that $f\left(x_{4}, b_{5}\right)=$ $f\left(x_{6}, b_{5}\right)$, then $\overline{G R}_{B \cup\left\{b_{5}\right\}}(X)\left(x_{4}\right)=\frac{3+1}{4+1}=\frac{4}{5}$ and $Y_{x_{4}}^{\vee *}=$ $\left\{y \in Y_{x_{4}}^{\vee} ; f\left(x_{4}, b_{5}\right)=f\left(y, b_{5}\right)\right\}=\left\{x_{6}\right\}$.

$\overline{G R}_{B \cup\left\{b_{5}\right\}}(X)\left(x_{5}\right):$ For any $y \in Y_{x_{5}}^{\vee}, f\left(x_{5}, b_{5}\right) \neq$ $f\left(y, b_{5}\right)$, so we have $\overline{G R}_{B \cup\left\{b_{5}\right\}}(X)\left(x_{5}\right)=\frac{3}{4+1}=\frac{3}{5}$ and $Y_{x_{5}}^{\vee *}=\left\{y ; \underset{y \in X}{\vee} G R_{B \cup\left\{b_{5}\right\}}\left(x_{5}, y\right)\right\}=\left\{x_{3}, x_{6}\right\}$.

$\overline{G R}_{B \cup\left\{b_{5}\right\}}(X)\left(x_{j}\right)=1, x_{j} \in\left\{x_{2}, x_{3}, x_{6}\right\}$.

\subsection{Incrementally updating approximations when removing an attribute}

Proposition 4 Let $S=(U, A, V, F)$ be an information system, $B \subseteq A, b \in B$. For any $x, y \in U$,

$$
\begin{aligned}
& G R_{B-\{b\}}(x, y)=\frac{|B| \cdot G R_{B}(x, y)-G R_{\{b\}}(x, y)}{|B|-1} \\
& = \begin{cases}\frac{|B| \cdot G R_{B}(x, y)+1}{|B|+1}, & f(x, b)=f(y, b) ; \\
\frac{|B| \cdot G R_{B}(x, y)}{|B|+1}, & f(x, b) \neq f(y, b) .\end{cases}
\end{aligned}
$$

Proof. This proof is straightforward.

Proposition 5 Let $S=(U, A, V, F)$ be an information system, $B \subseteq A, b \in B, X \subseteq U$. The lower and upper approximations of $X$ by removing $b$ from $B$ can be updated respectively as follows.

Lower approximation: If $x_{i} \in U-X$, then $\underline{G R}_{B-\{b\}}(X)\left(x_{i}\right)=\underline{G R}_{B}(X)\left(x_{i}\right)=0$; If $x_{i} \in X$, then $\underline{G R}_{B-\{b\}}(X)\left(x_{i}\right)$ 


$$
= \begin{cases}\frac{|B| G R_{B}(X)\left(x_{i}\right)-1}{|B|-1}, & \exists y \in Y_{x_{i}}^{\wedge}\left(f\left(x_{i}, b\right) \neq f(y, b)\right) ; \\ \frac{|B| G R_{B}(X)\left(x_{i}\right)}{|B|-1}, & \forall y\left(y \in Y_{x_{i}}^{\wedge} \rightarrow f\left(x_{i}, b\right)=f(y, b)\right) .\end{cases}
$$

Where $Y_{x_{i}}^{\wedge}=\left\{y \in U-X ; \underline{G R}_{B}(X)\left(x_{i}\right)=1-\right.$ $\left.G R_{B}\left(x_{i}, y\right)\right\}=\left\{y ; \underset{y \in U-X}{\vee} G R_{B}\left(x_{i}, y\right)\right\}$.

Upper approximation: If $x_{j} \in X$, then $\overline{G R}_{B-\{b\}}(X)\left(x_{j}\right)=\overline{G R}_{B}(X)\left(x_{j}\right)=1$; If $x_{j} \in U-X$, then $\overline{G R}_{B-\{b\}}(X)\left(x_{j}\right)$

$$
= \begin{cases}\frac{|B| \overline{G R}_{B}(X)\left(x_{j}\right)}{|B|-1}, & \exists y \in Y_{x_{j}}^{\vee}\left(f\left(x_{j}, b\right) \neq f(y, b)\right) ; \\ \frac{|B| \cdot G R_{B}(x, y)}{|B|-1}, & f(x, b) \neq f(y, b) .\end{cases}
$$

Where $Y_{x_{j}}^{\vee}=\left\{y \in X ; \overline{G R}_{B}(X)\left(x_{j}\right)=G R_{B}\left(x_{j}, y\right)\right\}=\{y$; $\left.\underset{y \in X}{\vee} G R_{B}\left(x_{j}, y\right)\right\}$.

Proof. Lower approximation: For any $x_{i} \in U-X$, it is obviously that $\underline{G R}_{B-\{b\}}(X)\left(x_{i}\right)=G R_{B}(X)\left(x_{i}\right)=0$.

For any $x_{i} \in X$, if $\exists y \in Y_{x_{i}}^{\wedge}$, such that $f\left(x_{i}, b\right) \neq$ $f(y, b)$, then $\underline{G R}_{B-\{b\}}(X)\left(x_{i}\right) \stackrel{=}{=} 1-G R_{B-\{b\}}\left(x_{i}, y\right)=$ $1-\frac{|B| G R_{B}\left(x_{i}, y\right)}{|B|-1}=1-\frac{|B|\left(1-\underline{G} R_{B}(X)\left(x_{i}\right)\right)}{|B|-1}=\frac{|B| G R_{B}(X)\left(x_{i}\right)-1}{|B|-1}$.

If $f\left(x_{i}, b\right)=f(y, b)$ for any $y \in Y_{x_{i}}^{\wedge}$, then $\underline{G R}_{B-\{b\}}(X)\left(x_{i}\right)=\hat{y}_{y \in U-X}\left(1-G R_{B-\{b\}}\left(x_{i}, y\right)\right)=1-$ $\left(G R_{B-\{b\}}\left(x_{i}, y_{0}\right) \vee\left(\underset{y \in U-X-Y_{x_{i}}^{\wedge}, f\left(x_{i}, b\right) \neq f(y, b)}{y \in U-X} G R_{B-\{b\}}\left(x_{i}, y\right)\right)\right)=$ $1-\left(\frac{|B| G R_{B}\left(x_{i}, y_{0}\right)-1}{|B|-1} \vee\left({ }_{y \in U-X-Y_{x_{i}}^{\wedge}, f\left(x_{i}, b\right) \neq f(y, b)} \frac{|B| G R_{B}\left(x_{i}, y\right)}{|B|-1}\right)\right.$, where $y_{0} \in Y_{x_{i}}^{\wedge}$. For any $y \in U-X-Y_{x_{i}}^{\wedge}$, we have $G R_{B}\left(x_{i}, y_{0}\right)>G R_{B}\left(x_{i}, y\right)$, that is $\frac{|B| G R_{B}\left(x_{i}, y_{0}\right)-1}{|B|-1} \geqslant$ $\frac{|B| G R_{B}\left(x_{i}, y\right)}{|B|-1}$. Therefore, $\quad \underline{G R}_{B-\{b\}}(X)\left(x_{i}\right)=1-$ $\frac{|B| G R_{B}\left(x_{i}, y_{0}\right)-1}{|B|-1}=\frac{|B| G R_{B}(X)\left(x_{i}\right)}{|B|-1}$.

Upper approximation: For any $x_{j} \in X$, it is obviously that $\overline{G R}_{B-\{b\}}(X)\left(x_{j}\right)=\overline{G R}_{B}(X)\left(x_{j}\right)=1$. For any $x_{j} \in U-X$, if $\exists y \in Y_{x_{j}}^{\vee}$ such that $f\left(x_{j}, b\right) \neq f(y, b)$, then $\overline{G R}_{B-\{b\}}(X)\left(x_{j}\right)=G R_{B-\{b\}}\left(x_{j}, y\right)=\frac{|B| G R_{B}\left(x_{j}, y\right)}{|B|-1}=$ $\frac{|B| G R_{B}(X)\left(x_{j}\right)}{|B|-1}$.

If $f\left(x_{j}, b\right)=f(y, b)$ for any $y \in Y_{x_{j}}^{\vee}$, then $\quad \overline{G R}_{B-\{b\}}(X)\left(x_{j}\right)=\underset{y \in X}{\vee} G R_{B-\{b\}}\left(x_{j}, y\right)=$ $G R_{B-\{b\}}\left(x_{j}, y_{0}\right) \vee\left(\underset{y \in X-Y_{x_{j}}^{\vee}, f\left(x_{j}, b\right) \neq f(y, b)}{\vee} G R_{B-\{b\}}\left(x_{j}, y\right)\right)=$ $\frac{|B| G R_{B}\left(x_{j}, y_{0}\right)-1}{|B|-1} \vee \quad\left(\underset{y \in X-Y_{x_{j}}^{\vee}, f\left(x_{j}, b\right) \neq f(y, b)}{\vee} \frac{|B| G R_{B}\left(x_{j}, y\right)}{|B|-1}\right)$, where $y_{0} \in Y_{x_{j}}^{\vee}$. For any $y \in X-Y_{x_{j}}^{\vee}$, we have $G R_{B}\left(x_{j}, y_{0}\right)>G R_{B}\left(x_{j}, y\right)$, that is $\frac{|B| G R_{B}\left(x_{j}, y_{0}\right)-1}{|B|-1} \geqslant$ $\frac{|B| G R_{B}\left(x_{j}, y\right)}{|B|-1}$. Therefore, $\quad \overline{G R}_{B-\{b\}}(X)\left(x_{j}\right)=$
$\frac{|B| G R_{B}\left(x_{j}, y_{0}\right)-1}{|B|-1}=\frac{|B| G R_{B}(X)\left(x_{j}\right)-1}{|B|-1}$.

Particularly, if $Y_{x_{i}}^{\wedge}=\left\{y_{k}\right\}$ and $Y_{x_{j}}^{\vee}=\left\{y_{l}\right\}$ are subsets of the universe with single element, the lower and upper approximations of $X$ by removing $b$ from $B$ can be updated respectively as follows.

Lower approximation: If $x_{i} \in U-X$, then $\underline{G R}_{B-\{b\}}(X)\left(x_{i}\right)=\underline{G R}_{B}(X)\left(x_{i}\right)=0$. If $x_{i} \in X$, then

$\underline{G R}_{B-\{b\}}(X)\left(x_{i}\right)= \begin{cases}\frac{|B| \underline{G R}_{B}(X)\left(x_{i}\right)-1}{|B|-1}, & f\left(x_{i}, b\right) \neq f\left(y_{k}, b\right) ; \\ \frac{|B| \underline{G} B}{|B|-1},\left(x_{i}\right) & f\left(x_{i}, b\right)=f\left(y_{k}, b\right) .\end{cases}$

Upper approximation: If $x_{j} \in X$, then $\overline{G R}_{B-\{b\}}(X)\left(x_{j}\right)=\overline{G R}_{B}(X)\left(x_{j}\right)=1$. If $x_{j} \in U-X$, then

$\overline{G R}_{B-\{b\}}(X)\left(x_{j}\right)= \begin{cases}\frac{|B| \overline{G R}_{B}(X)\left(x_{j}\right)}{|B|-1}, & f\left(x_{j}, b\right) \neq f\left(y_{l}, b\right) ; \\ \frac{|B| G R_{B}(X)\left(x_{j}\right)-1}{|B|-1}, & f\left(x_{j}, b\right)=f\left(y_{l}, b\right) .\end{cases}$

Proposition 6 The set of $Y_{x_{i}}^{\wedge}$ and $Y_{x_{j}}^{\vee}$ by removing $b$ from $B$ can be updated respectively as follows.

$$
\begin{aligned}
& Y_{x_{i}}^{\wedge *}=\left\{\begin{array}{l}
\left\{y \in Y_{x_{i}}^{\wedge} ; f\left(x_{i}, b\right)=f(y, b)\right\}, \exists y \in Y_{x_{i}}^{\wedge} \\
\left(f\left(x_{i}, b\right) \neq f(y, b)\right) ; \\
\left\{y ; \underset{y \in U-X}{\vee} G R_{B-\{b\}}\left(x_{i}, y\right)\right\}, \forall y\left(y \in Y_{x_{i}}^{\wedge}\right. \\
\left.f\left(x_{i}, b\right)=f(y, b)\right) .
\end{array}\right. \\
& Y_{x_{j}}^{\vee *}=\left\{\begin{array}{l}
\left\{y \in Y_{x_{j}}^{\vee} ; f\left(x_{j}, b\right)=f(y, b)\right\} ; \exists y \in Y_{x_{j}}^{\vee} \\
\left(f\left(x_{j}, b\right) \neq f(y, b)\right), \\
\left\{y ; \underset{y \in X}{\vee} G R_{B-\{b\}}\left(x_{j}, y\right)\right\} ; \forall y\left(y \in Y_{x_{j}}^{\vee} \rightarrow\right. \\
\left.f\left(x_{j}, b\right)=f(y, b)\right) .
\end{array}\right.
\end{aligned}
$$

Algorithm 3.2 (Incremental algorithm for updating approximations when removing an attribute $b$ )

Step 1: Input the relation matrix $M_{n \times m}$; $\underline{G R}_{B}(X)\left(x_{i}\right), Y_{x_{i}}^{\wedge}, x_{i} \in X ; \overline{G R}_{B}(X)\left(x_{j}, Y_{x_{j}}^{\vee}, x_{j} \in U-X\right.$.

Step 2: We get a new relation matrix $M_{n \times m}^{*}=$ $G R_{B-\{b\}}\left(x_{i}, x_{j}\right)= \begin{cases}\frac{|B| \cdot G R_{B}\left(x_{i}, x_{j}\right)+1}{|B|+1}, & f\left(x_{i}, b\right)=f\left(x_{j}, b\right) ; \\ \frac{|B| \cdot G R_{B}\left(x_{i}, x_{j}\right)}{|B|+1}, & f\left(x_{i}, b\right) \neq f\left(x_{j}, b\right) .\end{cases}$ $x_{i} \in X, x_{j} \in U-X$. // According to Proposition 4. $X$

Step 3: Calculate the lower approximations $x_{i} \in$

If $\exists y \in Y_{x_{i}}^{\wedge}\left(f\left(x_{i}, b\right) \neq f(y, b)\right.$, then $\underline{G R}_{B-\{b\}}(X)\left(x_{i}\right)=$ $\frac{|B| G R_{B}(X)\left(x_{i}\right)-1}{|B|-1}$ and $Y_{x_{i}}^{\wedge *}=\left\{y \in Y_{x_{i}}^{\wedge} ; f\left(x_{i}, b\right)=f(y, b)\right\}$. 
Else $\quad Y_{x_{i}}^{\wedge *}=\left\{y ; \underset{y \in U-X}{\vee} G R_{B-\{b\}}\left(x_{i}, y\right)\right\} \quad$ and $\underline{G R}_{B-\{b\}}(X)\left(x_{i}\right)=\frac{|B| G R_{B}(X)\left(x_{i}\right)}{|B|-1}$. // According to Proposition 5 and 6.

Step 4: Calculate the upper approximations $x_{j} \in$ $U-X$

If $\exists y \in Y_{x_{j}}^{\vee}\left(f\left(x_{j}, b\right) \neq f(y, b)\right), \quad$ then $\overline{G R}_{B-\{b\}}(X)\left(x_{j}\right)=\frac{|B| \overline{G R}_{B}(X)\left(x_{j}\right)}{|B|-1} \quad$ and $\quad Y_{x_{j}}^{\vee *}=\{y \in$ $\left.Y_{x_{j}}^{\vee} ; f\left(x_{j}, b\right)=f(y, b)\right\}$.

Else $\overline{G R}_{B-\{b\}}(X)\left(x_{j}\right)=\frac{|B| \overline{G R}_{B}(X)\left(x_{j}\right)-1}{|B|-1}$ and $Y_{x_{j}}^{\vee *}=$ $\left\{y ; \underset{y \in X}{\vee} G R_{B-\{b\}}\left(x_{j}, y\right)\right\}$. // According to Proposition 5 and 6.

Step 5: Output the relation matrix $M_{n \times m}^{*}$; $\underline{G R}_{B}(X)\left(x_{i}\right), Y_{x_{i}}^{\wedge}, x_{i} \in X ; \overline{G R}_{B}(X)\left(x_{j}, Y_{x_{j}}^{\vee}, x_{j} \in U-X ;\right.$ $\underline{G R}_{B}(X)\left(x_{i}\right)=0, x_{i} \in U-X ; \overline{G R}_{B}(X)\left(x_{j}=1, x_{j} \in X\right.$.

The Algorithm 3.2 has a time complexity of $O(|X||U-X|)$, which is mainly decided by Step 2 . In the following Example 3, We use the results from Example 2 to demonstrate how algorithm 3.2 update the approximations when removing an attribute.

Example 3 We consider the information system given in Table 1. Let $U=\left\{x_{1}, x_{2}, x_{3}, x_{4}, x_{5}, x_{6}\right\}$ be the universal set, $B=\left\{b_{1}, b_{2}, b_{3}, b_{4}, b_{5}\right\}$ be the conditional attribute set, $X=\left\{x_{2}, x_{3}, x_{6}\right\}$ be the decision set. Removing an attribute $b_{2}$ from $B$.

\begin{tabular}{cccccc}
\multicolumn{6}{c}{ Table 2.} \\
\hline & $b_{1}$ & $b_{3}$ & $b_{4}$ & $b_{5}$ & $d$ \\
\hline$x_{1}$ & 2 & 1 & 1 & 3 & $U-X$ \\
$x_{2}$ & 2 & 1 & 2 & 2 & $X$ \\
$x_{3}$ & 2 & 2 & 1 & 3 & $X$ \\
$x_{4}$ & 1 & 1 & 2 & 2 & $U-X$ \\
$x_{5}$ & 1 & 2 & 2 & 3 & $U-X$ \\
$x_{6}$ & 1 & 2 & 2 & 2 & $X$ \\
\hline
\end{tabular}

Using the result of Example 2.

$$
M_{3 \times 3}=x_{x_{3}} x_{2}\left(\begin{array}{ccc}
3 / 5 & x_{4} & x_{5} \\
x_{6} & 3 / 5 & 2 / 5 \\
4 / 5 & 0 & 3 / 5 \\
0 & 4 / 5 & 3 / 5
\end{array}\right)
$$

$\underline{G R}_{B}(X)\left(x_{2}\right)=\frac{2}{5}, Y_{x_{2}}^{\wedge}=\left\{x_{1}, x_{4}\right\} ; \underline{G R}_{B}(X)\left(x_{3}\right)=\frac{1}{5}$, $Y_{x_{3}}^{\wedge}=\left\{x_{1}\right\} ; \underline{G R}_{B}(X)\left(x_{6}\right)=\frac{1}{5}, Y_{x_{6}}^{\wedge}=\left\{x_{4}\right\} ; \underline{G R}_{B}(X)\left(x_{i}\right)=$ $0, x_{i} \in\left\{x_{1}, x_{4}, x_{5}\right\}$.
$\overline{G R}_{B}(X)\left(x_{1}\right)=\frac{4}{5}, \quad Y_{x_{1}}^{\vee}=\left\{x_{3}\right\} ; \quad \overline{G R}_{B}(X)\left(x_{4}\right)=$ $\frac{4}{5}, Y_{x_{4}}^{\vee}=\left\{x_{6}\right\} ; \quad \overline{G R}_{B}(X)\left(x_{5}\right)=\frac{3}{5}, \quad Y_{x_{5}}^{\vee}=\left\{x_{3}, x_{6}\right\} ;$ $\overline{G R}_{B}(X)\left(x_{j}\right)=1, x_{j} \in\left\{x_{2}, x_{3}, x_{6}\right\}$.

From the Step 2 of Algorithm 3.2 we have a new relation matrix $M_{3 \times 3}^{*}$

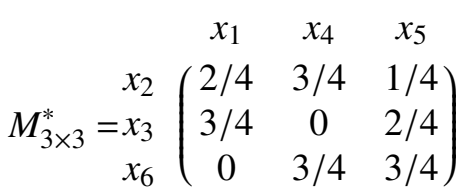

The lower and upper approximations of $X=$ $\left\{x_{2}, x_{3}, x_{6}\right\}$ by removing $b_{2}$ from $B$ are updated as follows.

From the step 3 of algorithm 3.2 we have the lower approximations:

$\underline{G R}_{B-\left\{b_{2}\right\}}(X)\left(x_{2}\right): \exists x_{4} \in Y_{x_{2}}^{\wedge}$, such that $f\left(x_{2}, b_{2}\right) \neq$ $f\left(x_{4}, b_{2}\right)$, then $\underline{G R}_{B-\left\{b_{2}\right\}}(X)\left(x_{2}\right)=\frac{2-1}{5-1}=\frac{1}{4}$ and $Y_{x_{2}}^{\wedge *}=$ $\left\{y \in Y_{x_{2}}^{\wedge} ; f\left(x, b_{2}\right) \neq f\left(y, b_{2}\right)\right\}=\left\{x_{4}\right\}$.

For any $y \in Y_{x_{3}}^{\wedge}, \quad f\left(x_{3}, b_{2}\right)=f\left(y, b_{2}\right)$, so we have $\underline{G R}_{B-\left\{b_{2}\right\}}(X)\left(x_{3}\right)=\frac{1}{5-1}=\frac{1}{4}$ and $Y_{x_{3}}^{\wedge *}=\left\{y ; \underset{y \in U-X}{\vee} G R_{B-\left\{b_{2}\right\}}\left(x_{3}, y\right)\right\}=\left\{x_{1}\right\}$.

For any $y \in Y_{x_{6}}^{\wedge}, \quad f\left(x_{6}, b_{2}\right)=f\left(y, b_{2}\right)$, so we have $\underline{G R}_{B-\left\{b_{2}\right\}}(X)\left(x_{6}\right)=\frac{1}{5-1}=\frac{1}{4}$ and $Y_{x_{6}}^{\wedge *}=\left\{y ; \underset{y \in U-X}{\vee} G R_{B-\left\{b_{2}\right\}}\left(x_{6}, y\right)\right\}=\left\{x_{4}, x_{5}\right\}$.

$$
\underline{G R}_{B-\left\{b_{2}\right\}}(X)\left(x_{i}\right)=0, x_{i} \in\left\{x_{1}, x_{4}, x_{5}\right\} .
$$

From the step 4 of algorithm 3.2 we have the upper approximations:

$\overline{G R}_{B-\left\{b_{2}\right\}}(X)\left(x_{1}\right)$ : For any $y \in Y_{x_{1}}^{\vee}, f\left(x_{1}, b_{2}\right)=$ $f\left(y, b_{2}\right)$, so we have $\overline{G R}_{B-\left\{b_{2}\right\}}(X)\left(x_{1}\right)=\frac{4-1}{5-1}=\frac{3}{4}$ and $Y_{x_{1}}^{\vee *}=\left\{y ; \underset{y \in X}{\vee} G R_{B-\left\{b_{2}\right\}}\left(x_{1}, y\right)\right\}=\left\{x_{3}\right\}$.

$\overline{G R}_{B-\left\{b_{2}\right\}}(X)\left(x_{4}\right)$ : For any $y \in Y_{x_{4}}^{\vee}, f\left(x_{4}, b_{2}\right)=$ $f\left(y, b_{2}\right)$, so we have $\overline{G R}_{B-\left\{b_{2}\right\}}(X)\left(x_{4}\right)=\frac{4-1}{5-1}=\frac{3}{4}$ and $Y_{x_{4}}^{\vee *}=\left\{y ; \underset{y \in X}{\vee} G R_{B-\left\{b_{2}\right\}}\left(x_{4}, y\right)\right\}=\left\{x_{2}, x_{6}\right\}$.

$\overline{G R}_{B-\left\{b_{2}\right\}}(X)\left(x_{5}\right): \exists x_{6} \in Y_{x_{5}}^{\vee}$, such that $f\left(x_{5}, b_{2}\right) \neq$ $f\left(x_{6}, b_{2}\right)$, then $\overline{G R}_{B-\left\{b_{2}\right\}}(X)\left(x_{5}\right)=\frac{3}{5-1}=\frac{3}{4}$ and $Y_{x_{5}}^{\vee *}=$ $\left\{y \in Y_{x_{5}}^{\vee} ; f\left(x_{5}, b_{2}\right) \neq f\left(y, b_{2}\right)\right\}=\left\{x_{6}\right\}$.

$$
\overline{G R}_{B-\left\{b_{2}\right\}}(X)\left(x_{j}\right)=1, x_{j} \in\left\{x_{2}, x_{3}, x_{6}\right\} .
$$




\section{Incrementally updating approximations while adding or removing an object}

In this section, we consider the problem of updating approximations based on the garde indiscernibility relation of a target concept in terms of adding or removing an object.

\subsection{Incrementally updating approximations when adding an object}

Proposition 7 Let $S=(U, A, V, F)$ be an information system, $B \subseteq A, x^{\vee} \notin U, X \subseteq U, U^{\vee}=U \cup\left\{x^{\vee}\right\}$. The lower and upper approximations of $X$ by adding $x^{\vee}$ to $U$ can be updated respectively as follows.

Lower approximation: When $x_{i} \in U^{\vee}-X$, we have $G R_{B}^{\vee}(X)\left(x_{i}\right)=0$. When $x_{i} \in X$, we have:

If $1-G R_{B}\left(x_{i}, x^{\vee}\right)>\underline{G R}_{B}(X)\left(x_{i}\right)$, then $\underline{G R}_{B}^{\vee}(X)\left(x_{i}\right)=\underline{G R}_{B}(X)\left(x_{i}\right)$ and $Y_{x_{i}}^{\wedge *}=\bar{Y}_{X_{i}}^{\wedge}$

If $1-\overline{G R}_{B}\left(x_{i}, x^{\vee}\right)=G R_{B}(X)\left(x_{i}\right)$, then $\underline{G R}_{B}^{\vee}(X)\left(x_{i}\right)=\underline{G R}_{B}(X)\left(x_{i}\right)$ and $Y_{x_{i}}^{\wedge *}=Y_{x_{i}}^{\wedge} \cup\left\{x^{\vee}\right\}$.

If $1-G_{B}\left(x_{i}, x^{\vee}\right)<\underline{G R}_{B}(X)\left(x_{i}\right)$, then $\underline{G R}_{B}^{\vee}(X)\left(x_{i}\right)=1-G R_{B}\left(x_{i}, x^{\vee}\right)$ and $Y_{x_{i}}^{\wedge *}=\left\{x^{\vee}\right\}$.

Upper approximation: If $x_{j} \in X, \overline{G R}_{B}^{\vee}(X)\left(x_{j}\right)=1$; If $x_{j} \in U^{\vee}-\left\{x^{\vee}\right\}-X$, then $\overline{G R}_{B}^{\vee}(X)\left(x_{j}\right)=\overline{G R}_{B}(X)\left(x_{j}\right)$ and $Y_{x_{j}}^{\vee *}=Y_{x_{j}}^{\vee}$. If $x_{j}=x^{\vee}$, then $\overline{G R}_{B}^{\vee}(X)\left(x^{\vee}\right)=$ $\underset{y \in X}{\vee} G R_{B}\left(x^{\vee}, y\right)$ and $Y_{x^{\vee}}^{\vee *}=\left\{y ; \underset{y \in X}{\vee} G R_{B}\left(x^{\vee}, y\right)\right\}$.

Proof. Lower approximation:

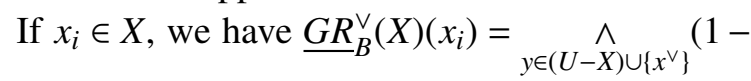
$\left.G R_{B}\left(x_{i}, y\right)\right)=\left(1-G R_{B}\left(x_{i}, x^{\vee}\right)\right) \wedge(\underset{y \in U-X}{\wedge}(1-$ $\left.\left.G R_{B}\left(x_{i}, y\right)\right)\right)=\left(1-G R_{B}\left(x_{i}, x^{\vee}\right)\right) \wedge \underline{G R}_{B}(X)\left(x_{i}\right) . \quad$ If $x \in U^{\vee}-X$, it is obviously that $\underline{G R}_{B}^{\vee}(X)\left(x_{i}\right)=$ $\underline{G R}_{B}(X)\left(x_{i}\right)=0$.

Upper approximation:

If $x_{j} \in X$, it is obviously that $\overline{G R}_{B}^{\vee}(X)\left(x_{j}\right)=$ $\overline{G R}_{B}(X)\left(x_{j}\right)=1$. If $x_{j} \in U^{\vee}-\left\{x^{\vee}\right\}-X$, we have $\overline{G R}_{B}^{\vee}(X)\left(x_{j}\right)=\vee_{y \in X} G R_{B}\left(x_{j}, y\right)=\overline{G R}_{B}(X)\left(x_{j}\right)$. If $x_{j}=x^{\vee}$, we have $\overline{G R}_{B}^{\vee}(X)\left(x_{j}\right)=\underset{y \in X}{\vee} G R_{B}\left(x_{j}, y\right)$.

Algorithm 4.1 (Incremental algorithm for updating approximations when adding an object $x^{\vee}$ to $U-X)$
Step 1: Input the relation matrix $M_{n \times m}$; $\underline{G R}_{B}(X)\left(x_{i}\right), Y_{x_{i}}^{\wedge}, x_{i} \in X ; \overline{G R}_{B}(X)\left(x_{j}, Y_{x_{j}}^{\vee}, x_{j} \in U-X\right.$; the increasing object $x^{\vee}$.

Step 2: We get a new relation matrix $M_{n \times(m+1)}^{*}$. // Calculate $G R_{B}\left(x^{\vee}, x_{i}\right), x_{i} \in X$. $X$

Step 3: Calculate the lower approximations $x_{i} \in$

If $1-G R_{B}\left(x_{i}, x^{\vee}\right)>\underline{G R}_{B}(X)\left(x_{i}\right), \quad$ then $\underline{G R}_{B}^{\vee}(X)\left(x_{i}\right)=\underline{G R}_{B}(X)\left(x_{i}\right)$ and $\underline{Y_{x_{i}}^{\wedge *}}=Y_{x_{i}}^{\wedge}$.

If $1-G R_{B}\left(x_{i}, x^{\vee}\right)=\underline{G R} R_{B}(X)\left(x_{i}\right)$, then $\underline{G R}_{B}^{\vee}(X)\left(x_{i}\right)=\underline{G R}_{B}(X)\left(x_{i}\right)$ and $Y_{x_{i}}^{\wedge *}=Y_{x_{i}}^{\wedge} \cup\left\{x^{\vee}\right\}$.

If $1-G R_{B}\left(x_{i}, x^{\vee}\right)<G R_{B}(X)\left(x_{i}\right)$, then $\underline{G R}_{B}^{\vee}(X)\left(x_{i}\right)=1-G R_{B}\left(x_{i}, x^{\vee}\right)$ and $Y_{x_{i}}^{\wedge *}=\left\{x^{\vee}\right\} . \quad / /$ According to Proposition 7.

Step 4: Calculate the upper approximations $x_{j} \in$ $U^{\vee}-X$

If $x_{j} \in U^{\vee}-\left\{x^{\vee}\right\}-X$, then $\overline{G R}_{B}^{\vee}(X)\left(x_{j}\right)=$ $\overline{G R}_{B}(X)\left(x_{j}\right)$ and $Y_{x_{j}}^{\vee *}=Y_{x_{j}}^{\vee}$.

$$
\overline{G R}_{B}^{\vee}(X)\left(x^{\vee}\right)=\underset{y \in X}{\vee} G R_{B}\left(x^{\vee}, y\right) \quad \text { and } \quad Y_{x \vee}^{\vee *}=
$$

$\left\{y ; \underset{y \in X}{\vee} G R_{B}\left(x^{\vee}, y\right)\right\}$. // According to Proposition 7.

Step 5: Output the relation matrix $M_{n \times(m+1)}^{*}$; $\underline{G R}_{B}^{\vee}(X)\left(x_{i}\right), Y_{x_{i}}^{\wedge *}, x_{i} \in X ; \overline{G R}_{B}^{\vee}(X)\left(x_{j}\right), Y_{x_{J}}^{\vee *}, x_{j} \in U^{\vee}-$ $X . \underline{G R}_{B}^{\vee}(X)\left(x_{i}\right)=0, x_{j} \in U^{\vee}-X, \overline{G R}_{B}^{\vee}(X)\left(x_{j}\right)=1$, $x_{j} \in X$.

The time complexity of Algorithm 4.1 is $O(|X||B|)$, which is mainly decided by Step 2. In the following Example 4, We use the results from Example 3 to demonstrate how algorithm 4.1 update the approximations when adding an object to $U-X$. Example 4 We consider the information system given in Table 2. Let $U=\left\{x_{1}, x_{2}, x_{3}, x_{4}, x_{5}, x_{6}\right\}$ be the universal set, $B=\left\{b_{1}, b_{3}, b_{4}, b_{5}\right\}$ be the conditional attribute set, $X=\left\{x_{2}, x_{3}, x_{6}\right\}$ be the decision set. Adding an object $x_{7} \in \Psi$ to $U-X, U^{\vee}=U \cup\left\{x_{7}\right\}$.

\begin{tabular}{cccccc}
\multicolumn{6}{c}{ Table 3.} \\
\hline & $b_{1}$ & $b_{3}$ & $b_{4}$ & $b_{5}$ & $d$ \\
\hline$x_{1}$ & 2 & 1 & 1 & 3 & $U-X$ \\
$x_{2}$ & 2 & 1 & 2 & 2 & $X$ \\
$x_{3}$ & 2 & 2 & 1 & 3 & $X$ \\
$x_{4}$ & 1 & 1 & 2 & 2 & $U-X$ \\
$x_{5}$ & 1 & 2 & 2 & 3 & $U-X$ \\
$x_{6}$ & 1 & 2 & 2 & 2 & $X$ \\
$x_{7}$ & 1 & 2 & 2 & 1 & $U-X$ \\
\hline
\end{tabular}


Using the result of Example 3.

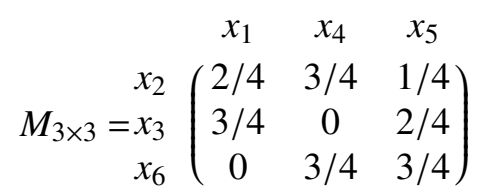

$\underline{G R}_{B}(X)\left(x_{2}\right)=\frac{1}{4}, Y_{x_{2}}^{\wedge}=\left\{x_{4}\right\} ; \underline{G R}_{B}(X)\left(x_{3}\right)=\frac{1}{4}, Y_{x_{3}}^{\wedge}=$ $\left\{x_{1}\right\} ; \underline{G R}_{B}(X)\left(x_{6}\right)=\frac{1}{4}, Y_{x_{6}}^{\wedge}=\left\{x_{4}, x_{5}\right\} ; \underline{G R}_{B}(X)\left(x_{i}\right)=$ $0, x_{i} \in\left\{x_{1}, x_{4}, x_{5}\right\}$.

$\overline{G R}_{B}(X)\left(x_{1}\right)=\frac{3}{4}, Y_{x_{1}}^{\vee}=\left\{x_{3}\right\} ; \quad \overline{G R}_{B}(X)\left(x_{4}\right)=$ $\frac{3}{4}, \quad Y_{x_{4}}^{\vee}=\left\{x_{2}, x_{6}\right\} ; \quad \overline{G R}_{B}(X)\left(x_{5}\right)=\frac{3}{4}, Y_{x_{5}}^{\vee}=\left\{x_{6}\right\} ;$ $\overline{G R}_{B}(X)\left(x_{j}\right)=1, x_{j} \in\left\{x_{2}, x_{3}, x_{6}\right\}$.

From the Step 2 of Algorithm 4.1 we have a new relation matrix:

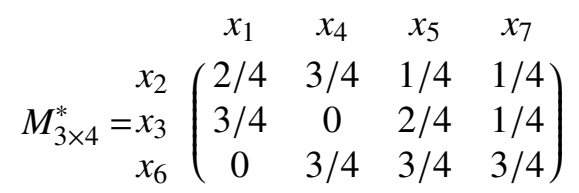

The lower and upper approximations of $X=$ $\left\{x_{2}, x_{3}, x_{6}\right\}$ by adding $x_{7}$ to $U-X$ are updated as follows.

From the step 3 of algorithm 4.1 we have the lower approximations:

$\underline{G R}_{B}^{\vee}(X)\left(x_{2}\right): \quad$ Since $1-G R_{B}\left(x_{2}, x_{7}\right)>$ $\underline{G R}_{B}(X)\left(x_{2}\right)$, then $\underline{G R}_{B}^{\vee}(X)\left(x_{2}\right)=\underline{G R}_{B}(X)\left(x_{2}\right)=\frac{1}{4}$ and $Y_{x_{2}}^{\wedge *}=Y_{x_{2}}^{\wedge}=\left\{x_{4}\right\}$.

$\underline{G R}_{B}^{\vee}(X)\left(x_{3}\right): \quad$ Since $1-G R_{B}\left(x_{3}, x_{7}\right)>$ $\underline{G R}_{B}(X)\left(x_{3}\right)$, then $\underline{G R}_{B}^{\vee}(X)\left(x_{3}\right)=\underline{G R}_{B}(X)\left(x_{3}\right)=\frac{1}{4}$ and $Y_{x_{3}}^{\wedge *}=Y_{x_{3}}^{\wedge}=\left\{x_{1}\right\}$.

$\underline{G R}_{B}^{\vee}(X)\left(x_{6}\right): \quad$ Since $1-G R_{B}\left(x_{6}, x_{7}\right)=$ $\underline{G R}_{B}(X)\left(x_{6}\right)$, then $\underline{G R}_{B}^{\vee}(X)\left(x_{6}\right)=\underline{G R}_{B}(X)\left(x_{6}\right)=\frac{1}{4}$ and $Y_{x_{6}}^{\wedge *}=Y_{x_{6}}^{\wedge} \cup\left\{x_{7}\right\}=\left\{x_{4}, x_{5}, x_{7}\right\}$.

$\underline{G R}_{B}^{\vee}(X)\left(x_{i}\right)=\underline{G R}_{B}(X)\left(x_{i}\right)=0, x_{i} \in\left\{x_{1}, x_{4}, x_{5}, x_{7}\right\}$.

From the step 4 of algorithm 4.1 we have the upper approximations:

$$
\overline{G R}_{B}^{\vee}(X)\left(x_{7}\right)=\underset{y \in\left\{x_{2}, x_{3}, x_{6}\right\}}{\vee} G R_{B}\left(x_{7}, y\right)=\frac{3}{4}, Y_{x_{7}}^{\vee *}=
$$

$\left\{y ; \underset{y \in\left\{x_{2}, x_{3}, x_{6}\right)}{\vee} G R_{B}\left(x_{7}, y\right)\right\}=\left\{x_{6}\right\}$.

$$
\overline{G R}_{B}^{\vee}(X)\left(x_{j}\right)=\overline{G R}_{B}(X)\left(x_{j}\right), Y_{x_{j}}^{\vee *}=Y_{x_{j}}^{\vee}, x_{j} \in
$$

$\left\{x_{1}, x_{4}, x_{5}\right\}$.

$$
\overline{G R}_{B}^{\vee}(X)\left(x_{j}\right)=1, x_{j} \in\left\{x_{2}, x_{3}, x_{6}\right\} \text {. }
$$

Proposition 8 Let $S=(U, A, V, F)$ be an information system, $B \subseteq A, x^{\vee} \notin U, X \subseteq U, U^{\vee}=U \cup\left\{x^{\vee}\right\}$.
The lower and upper approximations of $X^{\vee}=X \cup$ $\left\{x^{\vee}\right\}$ by adding $x^{\vee}$ to $X$ can be updated respectively as follows.

Lower approximation: If $x_{i} \in U^{\vee}-X^{\vee}$, then $G R_{B}^{\vee}\left(X^{\vee}\right)\left(x_{i}\right)=0$; If $x_{i} \in X$, then $\underline{G R}_{B}^{\vee}\left(X^{\vee}\right)\left(x_{i}\right)=$ $\underline{G R}_{B}(X)\left(x_{i}\right)$ and $Y_{x_{i}}^{\wedge *}=Y_{x_{i}}^{\wedge}$. If $x_{i}=x^{\vee}$, then $\underline{G R}_{B}^{\vee}\left(X^{\vee}\right)\left(x^{\vee}\right)=\hat{y}_{y \in U^{\vee}-X^{\vee}}^{\wedge}\left(1-G R_{B}\left(x^{\vee}, y\right)\right)$ and $Y_{x^{\vee}}^{\wedge *}=$ $\left\{y ; \underset{y \in U-X}{\vee} G R_{B}\left(x^{\vee}, y\right)\right\}$.

Upper approximation: When $x_{j} \in X^{\vee}$, we have $\overline{G R}_{B}^{\vee}\left(X^{\vee}\right)\left(x_{j}\right)=1$. When $x_{j} \in U^{\vee}-X^{\vee}$, we have:

If $G R_{B}\left(x_{j}, x^{\vee}\right)>\overline{G R}_{B}(X)\left(x_{j}\right)$, then $\overline{G R}_{B}^{\vee}\left(X^{\vee}\right)\left(x_{j}\right)=$ $G R_{B}\left(x_{j}, x^{\vee}\right)$ and $Y_{x_{j}}^{\vee *}=\left\{x^{\vee}\right\}$.

If $R_{B}\left(x_{j}, x^{\vee}\right)=\overline{G R}_{B}(X)\left(x_{j}\right)$, then $\overline{G R}_{B}^{\vee}\left(X^{\vee}\right)\left(x_{j}\right)=$ $\overline{G R}_{B}(X)\left(x_{j}\right)$ and $Y_{x_{j}}^{\vee *}=Y_{x_{j}}^{\vee} \cup\left\{x^{\vee}\right\}$.

If $R_{B}\left(x_{j}, x^{\vee}\right)<\overline{G R}_{B}(X)\left(x_{j}\right)$, then $\overline{G R}_{B}^{\vee}\left(X^{\vee}\right)\left(x_{j}\right)=$ $\overline{G R}_{B}(X)\left(x_{j}\right)$ and $Y_{x_{j}}^{\vee *}=Y_{x_{j}}^{\vee}$.

Proof. This proof is similar to that of Proposition 7.

Algorithm 4.2 (Incremental algorithm for updating approximations when adding an object $x^{\vee}$ to $X$ )

Step 1: Input the relation matrix $M_{n \times m}$; $\underline{G R}_{B}(X)\left(x_{i}\right), Y_{x_{i}}^{\wedge}, x_{i} \in X ; \overline{G R}_{B}(X)\left(x_{j}, Y_{x_{j}}^{\vee}, x_{j} \in U-X\right.$; the increasing object $x^{\vee}$.

Step 2: We get a new relation matrix $M_{(n+1) \times(m+1)}^{*}$. // Calculate $G R_{B}\left(x^{\vee}, x_{j}\right), x_{j} \in U-X$. $X^{\vee}$

Step 3: Calculate the lower approximations $x_{i} \in$

If $x_{i} \in X$, then $\underline{G R}_{B}^{\vee}\left(X^{\vee}\right)\left(x_{i}\right)=G R_{B}(X)\left(x_{i}\right)$ and $Y_{x_{i}}^{\wedge *}=Y_{x_{i}}^{\wedge}$. If $x_{i}=x^{\vee}$, then $\underline{G R}_{B}^{\vee}\left(X^{\vee}\right)\left(x^{\vee}\right)=$ $\hat{y}_{y \in U^{\vee}-X^{\vee}}^{\wedge}\left(1-G R_{B}\left(x^{\vee}, y\right)\right)$ and $Y_{x^{\vee}}^{\wedge *}=\left\{y ; \underset{y \in U-X}{\vee} G R_{B}\left(x^{\vee}, y\right)\right\}$. // According to Proposition 8.

Step 4: Calculate the upper approximations $x_{j} \in$ $U^{\vee}-X^{\vee}$

If $G R_{B}\left(x_{j}, x^{\vee}\right)>\overline{G R}_{B}(X)\left(x_{j}\right)$, then $\overline{G R}_{B}^{\vee}\left(X^{\vee}\right)\left(x_{j}\right)=$ $G R_{B}\left(x_{j}, x^{\vee}\right)$ and $Y_{x_{j}}^{\vee *}=\left\{x^{\vee}\right\}$.

If $G R_{B}\left(x_{j}, x^{\vee}\right)=\overline{G R}_{B}(X)\left(x_{j}\right)$, then $\overline{G R}_{B}^{\vee}\left(X^{\vee}\right)\left(x_{j}\right)=$ $\overline{G R}_{B}(X)\left(x_{j}\right)$ and $Y_{x_{j}}^{\vee *}=Y_{x_{j}}^{\vee} \cup\left\{x^{\vee}\right\}$.

If $G R_{B}\left(x_{j}, x^{\vee}\right)<\overline{G R}_{B}(X)\left(x_{j}\right)$, then $\overline{G R}_{B}^{\vee}\left(X^{\vee}\right)\left(x_{j}\right)=$ $\overline{G R}_{B}(X)\left(x_{j}\right)$ and $Y_{x_{j}}^{\vee *}=Y_{x_{j}}^{\vee}$. // According to Proposition 8 . 
Step 5: Output the relation matrix $M_{(n+1) \times m}^{*}$; $\underline{G R}_{B}^{\vee}\left(X^{\vee}\right)\left(x_{i}\right), \quad Y_{x_{i}}^{\wedge *}, \quad x_{i} \in X^{\vee} ; \quad \overline{G R}_{B}^{\vee}\left(X^{\vee}\right)\left(x_{j}\right), \quad Y_{x_{j}}^{\vee *}$, $x_{j} \in U^{\vee}-X^{\vee} ; \quad \underline{G R}_{B}^{\vee}\left(X^{\vee}\right)\left(x_{i}\right)=0, x_{i} \in U^{\vee}-$ $X^{\vee}, \overline{G R}_{B}^{\vee}\left(X^{\vee}\right)\left(x_{j}\right)=1, x_{j} \in X^{\vee}$.

The Algorithm 4.2 has a time complexity of $O(|U-X||B|)$, which is mainly decided by Step 2 . In the following Example 5, We use the results from Example 4 to demonstrate how algorithm 4.2 update the approximations when adding an object to $X$.

Example 5 We consider the information system given in Table 3. Let $U=\left\{x_{1}, x_{2}, x_{3}, x_{4}, x_{5}, x_{6}, x_{7}\right\}$ be the universal set, $B=\left\{b_{1}, b_{3}, b_{4}, b_{5}\right\}$ be the conditional attribute set, $X=\left\{x_{2}, x_{3}, x_{6}\right\}$ be the decision set. Adding $x_{8} \in \Phi$ to $X, X^{\vee}=X \cup\left\{x_{8}\right\}$.

\begin{tabular}{cccccc}
\multicolumn{7}{c}{ Table 4. } \\
\hline & $b_{1}$ & $b_{3}$ & $b_{4}$ & $b_{5}$ & $d$ \\
\hline$x_{1}$ & 2 & 1 & 1 & 3 & $U-X$ \\
$x_{2}$ & 2 & 1 & 2 & 2 & $X$ \\
$x_{3}$ & 2 & 2 & 1 & 3 & $X$ \\
$x_{4}$ & 1 & 1 & 2 & 2 & $U-X$ \\
$x_{5}$ & 1 & 2 & 2 & 3 & $U-X$ \\
$x_{6}$ & 1 & 2 & 2 & 2 & $X$ \\
$x_{7}$ & 1 & 2 & 2 & 1 & $U-X$ \\
$x_{8}$ & 2 & 1 & 2 & 2 & $X$ \\
\hline
\end{tabular}

Using the result of Example 4.

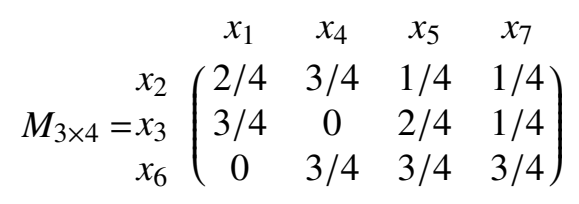

$\underline{G R}_{B}(X)\left(x_{2}\right)=\frac{1}{4}, \quad Y_{x_{2}}^{\wedge}=\left\{x_{4}\right\} ; \quad \underline{G R}_{B}(X)\left(x_{3}\right)=$ $\frac{1}{4}, Y_{x_{3}}^{\wedge}=\left\{x_{1}\right\} ; \quad \underline{G R}_{B}(X)\left(x_{6}\right)=\frac{1}{4}, Y_{x_{6}}^{\wedge}=\left\{x_{4}, x_{5}, x_{6}\right\} ;$ $\underline{G R}_{B}(X)\left(x_{i}\right)=0, x_{i} \in\left\{x_{1}, x_{4}, x_{5}, x_{7}\right\}$.

$\overline{G R}_{B}(X)\left(x_{1}\right)=\frac{3}{4}, \quad Y_{x_{1}}^{\vee}=\left\{x_{3}\right\} ; \quad \overline{G R}_{B}(X)\left(x_{4}\right)=$ $\frac{3}{4}, Y_{x_{4}}^{\vee}=\left\{x_{2}, x_{6}\right\} ; \quad \overline{G R}_{B}(X)\left(x_{5}\right)=\frac{3}{4}, Y_{x_{5}}^{\vee}=\left\{x_{6}\right\} ;$ $\overline{G R}_{B}(X)\left(x_{7}\right)=\frac{3}{4}, Y_{x_{5}}^{\vee}=\left\{x_{6}\right\} ; \overline{G R}_{B}(X)\left(x_{j}\right)=1, x_{j} \in$ $\left\{x_{2}, x_{3}, x_{6}\right\}$.
From the Step 2 of Algorithm 4.2 we have a new relation matrix $M_{4 \times 4}^{*}$ :

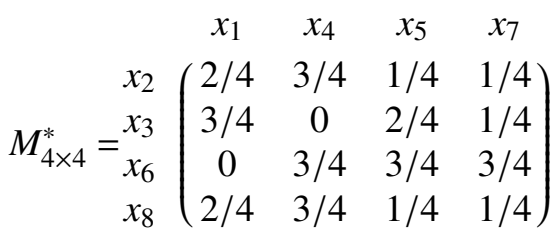

The lower and upper approximations of $X^{\vee}=$ $\left\{x_{2}, x_{3}, x_{6}, x_{8}\right\}$ by adding $x_{8}$ to $X$ are updated as follows.

From the step 3 of algorithm 4.2 we have the lower approximations:

$$
\begin{aligned}
& \quad \underline{G R}_{B}^{\vee}\left(X^{\vee}\right)\left(x_{8}\right)=\underset{y \in\left\{x_{1}, x_{4}, x_{5}, x_{7}\right\}}{\wedge}\left(1-G R_{B}\left(x_{8}, y\right)\right)=\frac{1}{4}, \\
& \text { and } Y_{x_{8}}^{\wedge *}=\left\{y ; \underset{y \in\left\{x_{1}, x_{4}, x_{5}, x_{7}\right\}}{\left.G R_{B}\left(x_{8}, y\right)\right\}=\left\{x_{4}\right\} .}\right. \\
& \quad \underline{G R}_{B}^{\vee}\left(X^{\vee}\right)\left(x_{i}\right)=\underline{G R}_{B}(X)\left(x_{i}\right), \quad Y_{x_{i}}^{\wedge *}=Y_{x_{i}}^{\wedge}, x_{i} \in \\
& \left\{x_{2}, x_{3}, x_{6}\right\} . \\
& \quad \underline{G R}_{B}^{\vee}\left(X^{\vee}\right)\left(x_{i}\right)=0, x_{i} \in\left\{x_{1}, x_{4}, x_{5}, x_{7}\right\} .
\end{aligned}
$$

From the step 4 of algorithm 4.2 we have the upper approximations:

$\overline{G R}_{B}^{\vee}\left(X^{\vee}\right)\left(x_{1}\right)$ : Since $G R_{B}\left(x_{1}, x_{8}\right)<\overline{G R}_{B}(X)\left(x_{1}\right)$, then $\overline{G R}_{B}^{\vee}\left(X^{\vee}\right)\left(x_{1}\right)=\overline{G R}_{B}(X)\left(x_{1}\right)=\frac{3}{4}$ and $Y_{x_{1}}^{\vee *}=$ $Y_{x_{1}}^{\vee}=\left\{x_{3}\right\}$.

$\overline{G R}_{B}^{\vee}\left(X^{\vee}\right)\left(x_{4}\right)$ : Since $G R_{B}\left(x_{4}, x_{8}\right)=\overline{G R}_{B}(X)\left(x_{4}\right)$, then $\overline{G R}_{B}^{\vee}\left(X^{\vee}\right)\left(x_{4}\right)=\overline{G R}_{B}(X)\left(x_{4}\right)=\frac{3}{4}$ and $Y_{x_{4}}^{\vee *}=$ $Y_{x_{4}}^{\vee} \cup\left\{x_{8}\right\}=\left\{x_{2}, x_{6}, x_{8}\right\}$.

$\overline{G R}_{B}^{\vee}\left(X^{\vee}\right)\left(x_{5}\right)$ : Since $G R_{B}\left(x_{5}, x_{8}\right)<\overline{G R}_{B}(X)\left(x_{5}\right)$, then $\overline{G R}_{B}^{\vee}\left(X^{\vee}\right)\left(x_{5}\right)=\overline{G R}_{B}(X)\left(x_{5}\right)=\frac{3}{4}$ and $Y_{x_{5}}^{\vee *}=$ $Y_{x_{5}}^{\vee}=\left\{x_{6}\right\}$.

$\overline{G R}_{B}^{\vee}\left(X^{\vee}\right)\left(x_{7}\right)$ : Since $G R_{B}\left(x_{7}, x_{8}\right)<\overline{G R}_{B}(X)\left(x_{7}\right)$, then $\overline{G R}_{B}^{\vee}\left(X^{\vee}\right)\left(x_{7}\right)=\overline{G R}_{B}(X)\left(x_{7}\right)=\frac{3}{4}$ and $Y_{x_{7}}^{\vee *}=$ $Y_{x_{7}}^{\vee}=\left\{x_{6}\right\}$.

$$
\overline{G R}_{B}^{\vee}\left(X^{\vee}\right)\left(x_{j}\right)=1, x_{j} \in\left\{x_{2}, x_{3}, x_{6}, x_{8}\right\} .
$$

\subsection{Incrementally updating approximations when removing an object}

Proposition 9 Let $S=(U, A, V, F)$ be an information system, $B \subseteq A, x^{\wedge} \in U$ and $x^{\wedge} \notin X, X \subseteq U$, $U^{\wedge}=U-\left\{x^{\wedge}\right\}$. The lower and upper approximations of $X$ by removing $x^{\wedge}$ from $U$ can be updated respectively as follows. 
Lower approximation: If $x_{i} \in U^{\wedge}-X$, then $\underline{G R}_{B}^{\wedge}(X)\left(x_{i}\right)=\underline{G R}_{B}(X)\left(x_{i}\right)=0$. If $x_{i} \in X$, then we have:

If $\underline{G R}_{B}(X)\left(x_{i}\right)=1-G R_{B}\left(x_{i}, x^{\wedge}\right)$, then $\underline{G R}_{B}^{\wedge}(X)\left(x_{i}\right)=\underset{y \in U^{\wedge}-X}{\wedge}\left(1-G R_{B}\left(x_{i}, y\right)\right)$ and $Y_{x_{i}}^{\wedge *}=$ $\left\{y ; \underset{y \in U^{\wedge}-X}{\vee} G R_{B}\left(x_{i}, y\right)\right\}$.

If $\exists y \in Y_{x_{i}}^{\wedge}$, such that $\underline{G R}_{B}(X)\left(x_{i}\right)=1-G R_{B}\left(x_{i}, y\right)$, then $\underline{G R}_{B}^{\wedge}(X)\left(x_{i}\right)=\underline{G R}_{B}(X)\left(x_{i}\right)$ and $Y_{x_{i}}^{\wedge *}=Y_{x_{i}}^{\wedge}-\left\{x^{\wedge}\right\}$.

Upper approximation: $\overline{G R}_{B}^{\wedge}(X)\left(x_{j}\right)=1, x_{j} \in X$, and $\overline{G R}_{B}^{\wedge}(X)\left(x_{j}\right)=\overline{G R}_{B}(X)\left(x_{j}\right), Y_{x_{i}}^{\vee *}=Y_{x_{i}}^{\vee}, x_{j} \in U^{\wedge}-$ $X$.

Algorithm 4.3 (Incremental algorithm for updating approximations when removing an object $x^{\wedge}$ from $U-X)$

Step 1: Input the relation matrix $M_{n \times m}$; $\underline{G R}_{B}(X)\left(x_{i}\right), Y_{x_{i}}^{\wedge}, x_{i} \in X ; \overline{G R}_{B}(X)\left(x_{j}, Y_{x_{j}}^{\vee}, x_{j} \in U-X\right.$.

Step 2: We get a new relation matrix $M_{n \times(m-1)}^{*}$. // Delete $G R_{B}\left(x^{\wedge}, x_{i}\right), x_{i} \in X$ from the relation matrix $M_{n \times m}$.

Step 3: Calculate the lower approximations $x_{i} \in$ $X$

If $\exists y \in Y_{x_{i}}^{\wedge}$, such that $\underline{G R}_{B}(X)\left(x_{i}\right)=1-G R_{B}\left(x_{i}, y\right)$, then $\underline{G R}_{B}^{\wedge}(X)\left(x_{i}\right)=\underline{G R}_{B}(X)\left(x_{i}\right)$ and $Y_{x_{i}}^{\wedge *}=Y_{x_{i}}^{\wedge}-\left\{x^{\wedge}\right\}$.

Else $\underline{G R_{B}^{\wedge}}(X)\left(x_{i}\right)=\wedge_{y \in U^{\wedge}-X}^{\wedge}\left(1-G R_{B}\left(x_{i}, y\right)\right)$ and $Y_{x_{i}}^{\wedge *}=\left\{y ; \underset{y \in U^{\wedge}-X}{\vee} G R_{B}\left(x_{i}, y\right)\right\} . \quad / /$ According to the Proposition 9.

Step 4: Calculate the upper approximations $x_{j} \in$ $U^{\wedge}-X$

$\overline{G R}_{B}^{\wedge}(X)\left(x_{j}\right)=\overline{G R}_{B}(X)\left(x_{j}\right), Y_{x_{i}}^{\vee *}=Y_{x_{i}}^{\vee} \cdot / /$ According to the Proposition 9.

Step 5: Output the relation matrix $M_{n \times(m-1)}^{*}$; $\underline{G R}_{B}^{\wedge}(X)\left(x_{i}\right), Y_{x_{i}}^{\wedge *}, x_{i} \in X ; \overline{G R}_{B}^{\wedge}(X)\left(x_{j}\right), Y_{x_{i} *}^{\vee *}, x_{j} \in U^{\wedge}-$ $X ; \underline{G R}_{B}^{\wedge}(X)\left(x_{i}\right)=0, x_{i} \in U^{\wedge}-X$ and $\overline{G R}_{B}^{\wedge}(X)\left(x_{j}\right)=$ $1, x_{j} \in X$.

The Algorithm 4.3 has a time complexity of $O(|X|)$, which is mainly decided by Step 2 . In the following Example 6, We use the results from Example 5 to demonstrate how algorithm 4.3 update the approximations when removing an object from $U-X$.

Example 6 We consider the information system given in Table 4. Let $U=\left\{x_{1}, x_{2}, x_{3}, x_{4}, x_{5}, x_{6}, x_{7}, x_{8}\right\}$ be the universal set, $B=\left\{b_{1}, b_{3}, b_{4}, b_{5}\right\}$ be the condi- tional attribute set, $X=\left\{x_{2}, x_{3}, x_{6}, x_{8}\right\}$ be the decision set. Removing $x_{4} \in \Psi$ from $U-X$.

\begin{tabular}{cccccc}
\multicolumn{6}{c}{ Table 5.} \\
\hline & $b_{1}$ & $b_{3}$ & $b_{4}$ & $b_{5}$ & $d$ \\
\hline$x_{1}$ & 2 & 1 & 1 & 3 & $U-X$ \\
$x_{2}$ & 2 & 1 & 2 & 2 & $X$ \\
$x_{3}$ & 2 & 2 & 1 & 3 & $X$ \\
$x_{5}$ & 1 & 2 & 2 & 3 & $U-X$ \\
$x_{6}$ & 1 & 2 & 2 & 2 & $X$ \\
$x_{7}$ & 1 & 2 & 2 & 1 & $U-X$ \\
$x_{8}$ & 2 & 1 & 2 & 2 & $X$ \\
\hline
\end{tabular}

Using the result of Example 5.

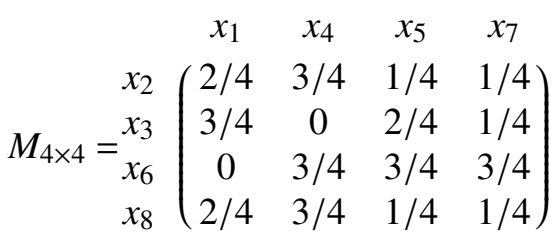

$\underline{G R}_{B}(X)\left(x_{2}\right)=\frac{1}{4}, \quad Y_{x_{2}}^{\wedge}=\left\{x_{4}\right\} ; \quad \underline{G R}_{B}(X)\left(x_{3}\right)=$ $\frac{1}{4}, Y_{x_{3}}^{\wedge}=\left\{x_{1}\right\} ; \quad \underline{G R}_{B}(X)\left(x_{6}\right)=\frac{1}{4}, Y_{x_{6}}^{\wedge}=\left\{x_{4}, x_{5}, x_{7}\right\} ;$ $\underline{G R}_{B}(X)\left(x_{8}\right)=\frac{1}{4}, Y_{x_{8}}^{\wedge}=\left\{x_{4}\right\} ; \underline{G R}_{B}(X)\left(x_{i}\right)=0, x_{i} \in$ $\left\{x_{1}, x_{4}, x_{5}, x_{7}\right\}$.

$\overline{G R}_{B}(X)\left(x_{1}\right)=\frac{3}{4}, \quad Y_{x_{1}}^{\vee}=\left\{x_{3}\right\} ; \quad \overline{G R}_{B}(X)\left(x_{4}\right)=$ $\frac{3}{4}, Y_{x_{4}}^{\vee}=\left\{x_{2}, x_{6}, x_{8}\right\} ; \quad \overline{G R}_{B}(X)\left(x_{5}\right)=\frac{3}{4}, Y_{x_{5}}^{\vee}=\left\{x_{6}\right\} ;$ $\overline{G R}_{B}(X)\left(x_{7}\right)=\frac{3}{4}, Y_{x_{7}}^{\vee}=\left\{x_{6}\right\} ; \overline{G R}_{B}(X)\left(x_{j}\right)=1, x_{j} \in$ $\left\{x_{2}, x_{3}, x_{6}, x_{8}\right\}$.

From the Step 2 of Algorithm 4.3 we have a new relation matrix $M_{4 \times 3}^{*}$ :

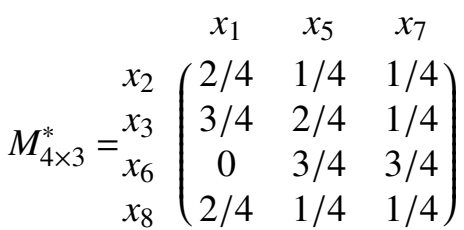

The lower and upper approximations of $X=$ $\left\{x_{2}, x_{3}, x_{6}, x_{8}\right\}$ by removing $x_{4}$ from $U-X$ be updated as follows:

From the step 3 of algorithm 4.3 we have the lower approximations:

$\underline{G R}_{B}^{\wedge}(X)\left(x_{2}\right): \quad$ Since $\quad \underline{G R}_{B}(X)\left(x_{2}\right)=1-$ $G R_{B}\left(x_{2}, x_{4}\right)$, then $\underline{G R}_{B}^{\wedge}(X)\left(x_{2}\right)=\wedge_{y \in\left\{x_{1}, x_{5}, x_{7}\right\}}^{\wedge}(1-$ $\left.G R_{B}\left(x_{2}, y\right)\right)=\frac{2}{4}$ and $Y_{x_{2}}^{\wedge *}=\left\{y ; \underset{y \in\left\{x_{1}, x_{5}, x_{7}\right\}}{\vee} G R_{B}\left(x_{2}, y\right)\right\}=$ $\left\{x_{1}\right\}$. 
$\underline{G R}_{B}^{\wedge}(X)\left(x_{3}\right):$ Since $Y_{x_{3}}^{\wedge}=\left\{x_{1}\right\}, \underline{G R}_{B}(X)\left(x_{3}\right)=1-$ $G R_{B}\left(x_{3}, x_{1}\right)$, then $\underline{G R}_{B}^{\wedge}(X)\left(x_{3}\right)=\underline{G R} \underline{R}_{B}(X)\left(x_{3}\right)=\frac{1}{4}$ and $Y_{x_{3}}^{\wedge *}=Y_{x_{3}}^{\wedge}-\left\{x_{4}\right\}=\left\{x_{1}\right\}$.

$\underline{G R}_{B}^{\wedge}(X)\left(x_{6}\right):$ Since $\exists x_{5} \in Y_{x_{6}}^{\wedge}$, such that $\underline{G R} R_{B}(X)\left(x_{5}\right)=1-G R_{B}\left(x_{6}, x_{5}\right)$, then $\underline{G R}_{B}^{\wedge}(X)\left(x_{6}\right)=$ $\underline{G R}_{B}(X)\left(x_{6}\right)=\frac{1}{4}$ and $Y_{x_{6}}^{\wedge *}=Y_{x_{6}}^{\wedge}-\left\{x_{4}\right\}=\left\{x_{5}, x_{7}\right\}$.

$\underline{G R}_{B}^{\wedge}(X)\left(x_{8}\right): \quad$ Since $\quad \underline{G R}_{B}(X)\left(x_{8}\right)=1-$ $G R_{B}\left(x_{8}, x_{4}\right)$, then $\underline{G R}_{B}^{\wedge}(X)\left(\overline{\left.x_{8}\right)}=\wedge_{y \in\left\{x_{1}, x_{5}, x_{7}\right\}}(1-\right.$ $\left.G R_{B}\left(x_{8}, y\right)\right)=\frac{2}{4}$ and $Y_{x_{8}}^{\wedge *}=\left\{y ; \underset{y \in\left\{x_{1}, x_{5}, x_{7}\right\}}{\vee} G R_{B}\left(x_{8}, y\right)\right\}=$ $\left\{x_{1}\right\}$.

$$
\underline{G R}_{B}^{\wedge}(X)\left(x_{i}\right)=\underline{G R}_{B}(X)\left(x_{i}\right)=0, x_{i} \in\left\{x_{1}, x_{5}, x_{7}\right\} .
$$

From the step 4 of algorithm 4.3 we have the upper approximations:

$$
\overline{G R}_{B}^{\wedge}(X)\left(x_{j}\right)=\overline{G R}_{B}(X)\left(x_{j}\right), \quad Y_{x_{i}}^{\vee *}=Y_{x_{i}}^{\vee}, \quad x_{j} \in
$$
$\left\{x_{1}, x_{5}, x_{7}\right\}$, and $\overline{G R} \wedge(X)\left(x_{j}\right)=1, x_{j} \in\left\{x_{2}, x_{3}, x_{6}, x_{8}\right\}$.

Proposition 10 Let $S=(U, A, V, F)$ be an information system, $B \subseteq A, U^{\wedge}=U-\left\{x^{\wedge}\right\}, x^{\wedge} \in X, X \subseteq U$. The lower and upper approximations of $X^{\wedge}=X-$ $\left\{x^{\wedge}\right\}$ by removing $x^{\wedge}$ from $X$ can be respectively updated as follows.

Lower approximation: $G R_{B}^{\wedge}\left(X^{\wedge}\right)\left(x_{i}\right)=0, x_{i} \in$ $U^{\wedge}-X^{\wedge}$, and $\underline{G R}_{B}^{\wedge}\left(X^{\wedge}\right)\left(x_{i}\right)=\underline{G R}_{B}(X)\left(x_{i}\right), Y_{x_{i}}^{\wedge *}=Y_{x_{i}}^{\wedge}$, $x_{i} \in X^{\wedge}$.

Upper approximation: If $x_{j} \in X^{\wedge}$, then $\overline{G R}_{B}^{\wedge}\left(X^{\wedge}\right)\left(x_{j}\right)=\overline{G R}_{B}(X)\left(x_{j}\right)=1$. If $x_{j} \in U^{\wedge}-X^{\wedge}$, we have:

If $\overline{G R}_{B}(X)\left(x_{j}\right)=G R_{B}\left(x_{j}, x^{\wedge}\right)$, then $\overline{G R}_{B}^{\wedge}\left(X^{\wedge}\right)\left(x_{j}\right)=$ $\underset{y \in X^{\wedge}}{\vee} G R_{B}\left(x_{j}, y\right)$ and $Y_{x_{j}}^{\vee *}=\left\{y ; \underset{y \in X^{\wedge}}{\vee} G R_{B}\left(x_{j}, y\right)\right\}$.

If $\exists y \in Y_{x_{j}}^{\vee}$, such that $\overline{G R}_{B}(X)\left(x_{j}\right)=G R_{B}\left(x_{j}, y\right) \neq$ $G R_{B}\left(x_{j}, x^{\wedge}\right)$, then $\overline{G R}_{B}^{\wedge}\left(X^{\wedge}\right)\left(x_{j}\right)=\overline{G R}_{B}(X)\left(x_{j}\right)$ and $Y_{x_{j}}^{\vee *}=Y_{x_{j}}^{\vee}-\left\{x^{\wedge}\right\}$.

Algorithm 4.4 (Incremental algorithm for updating approximations when removing an object $x^{\wedge}$ from $X$ )

Step 1: Input the relation matrix $M_{n \times m}$; $\underline{G R}_{B}(X)\left(x_{i}\right), Y_{x_{i}}^{\wedge}, x_{i} \in X ; \overline{G R}_{B}(X)\left(x_{j}, Y_{x_{j}}^{\vee}, x_{j} \in U-X\right.$.

Step 2: We get a new relation matrix $M_{(n-1) \times m}^{*}$. // Delete $G R_{B}\left(x^{\wedge}, x_{j}\right), x_{j} \in U-X$ from the relation matrix $M_{n} \times m$.

Step 3: Calculate the lower approximations $x_{i} \in$ $X^{\wedge}$

$\underline{G R_{B}^{\wedge}}\left(X^{\wedge}\right)\left(x_{i}\right)=\underline{G R}_{B}(X)\left(x_{i}\right), Y_{x_{i}}^{\wedge *}=Y_{x_{i}}^{\wedge} . / /$ According to the Proposition 10.
Step 4: Calculate the upper approximations $x_{j} \in$ $U^{\wedge}-X^{\wedge}$

If $\exists y \in Y_{x_{j}}^{\vee}$, such that $\overline{G R}_{B}(X)\left(x_{j}\right)=G R_{B}\left(x_{j}, y\right) \neq$ $G R_{B}\left(x_{j}, x^{\wedge}\right)$, then $\overline{G R}_{B}^{\wedge}\left(X^{\wedge}\right)\left(x_{j}\right)=\overline{G R}_{B}(X)\left(x_{j}\right)$ and $Y_{x_{j}}^{\vee *}=Y_{x_{j}}^{\vee}-\left\{x^{\wedge}\right\}$.

Else $\overline{G R}_{B}^{\wedge}\left(X^{\wedge}\right)\left(x_{j}\right)=\underset{y \in X^{\wedge}}{\vee} G R_{B}\left(x_{j}, y\right)$ and $Y_{x_{j}}^{\vee^{*}}=$ $\left\{y ; \underset{y \in X^{\wedge}}{\vee} G R_{B}\left(x_{j}, y\right)\right\}$. // According to the Proposition 10.

Step 5: Output the relation matrix $M_{(n-1) \times m}^{*}$; $\underline{G R}_{B}^{\wedge}\left(X^{\wedge}\right)\left(x_{i}\right), \quad Y_{x_{i} *}^{\wedge *} \quad x_{i} \in X^{\wedge} ; \quad \overline{G R}_{B}^{\wedge}\left(X^{\wedge}\right)\left(x_{j}\right), \quad Y_{x_{j} *}^{\vee *}$, $x_{j} \in U^{\wedge}-X^{\wedge} ; \underline{G R_{B}^{\wedge}}\left(X^{\wedge}\right)\left(x_{i}\right)=0, x_{i} \in U^{\wedge}-X^{\wedge}$, and $\overline{G R}_{B}^{\wedge}\left(X^{\wedge}\right)\left(x_{j}\right)=\overline{G R}_{B}(X)\left(x_{j}\right)=1, x_{j} \in X^{\wedge}$.

The Algorithm 4.4 has a time complexity of $O(|U-X|)$, which is mainly decided by Step 2 . In the following Example 7, We use the results from Example 6 to demonstrate how algorithm 4.4 update the approximations when removing an object from $X$.

Example 7 We consider the information system given in Table 5. Let $U=\left\{x_{1}, x_{2}, x_{3}, x_{5}, x_{6}, x_{7}, x_{8}\right\}$ be the universal set, $B=\left\{b_{1}, b_{3}, b_{4}, b_{5}\right\}$ be the conditional attribute set, $X=\left\{x_{2}, x_{3}, x_{6}, x_{8}\right\}$ be the decision set. Removing $x_{3} \in \Phi$ from $X, X^{\wedge}=X-\left\{x_{3}\right\}$.

\begin{tabular}{cccccc}
\multicolumn{6}{c}{ Table 6.} \\
\hline & $b_{1}$ & $b_{3}$ & $b_{4}$ & $b_{5}$ & $d$ \\
\hline$x_{1}$ & 2 & 1 & 1 & 3 & $U-X$ \\
$x_{2}$ & 2 & 1 & 2 & 2 & $X$ \\
$x_{5}$ & 1 & 2 & 2 & 3 & $U-X$ \\
$x_{6}$ & 1 & 2 & 2 & 2 & $X$ \\
$x_{7}$ & 1 & 2 & 2 & 1 & $U-X$ \\
$x_{8}$ & 2 & 1 & 2 & 2 & $X$ \\
\hline
\end{tabular}

Using the result of Example 6.

$$
M_{4 \times 3}=\begin{gathered}
x_{1} \\
x_{2} \\
x_{3} \\
x_{6} \\
x_{8}
\end{gathered}\left(\begin{array}{ccc}
2 / 4 & 1 / 4 & x_{7} \\
3 / 4 & 2 / 4 & 1 / 4 \\
0 & 3 / 4 & 3 / 4 \\
2 / 4 & 1 / 4 & 1 / 4
\end{array}\right)
$$

$\underline{G R}_{B}(X)\left(x_{2}\right)=\frac{2}{4}, Y_{x_{2}}^{\wedge}=\left\{x_{1}\right\} ; \underline{G R}_{B}(X)\left(x_{3}\right)=\frac{1}{4}, Y_{x_{3}}^{\wedge}=$ $\left\{x_{1}\right\} ; \underline{G R}_{B}(X)\left(x_{6}\right)=\frac{1}{4}, Y_{x_{6}}^{\wedge}=\left\{x_{5}, x_{7}\right\} ; \underline{G R}_{B}(X)\left(x_{8}\right)=$ $\frac{2}{4}, Y_{x_{8}}^{\wedge}=\left\{x_{1}\right\} ; \underline{G R}_{B}(X)\left(x_{i}\right)=0, x_{i} \in\left\{x_{1}, x_{5}, x_{7}\right\}$. 
$\overline{G R}_{B}(X)\left(x_{1}\right)=\frac{3}{4}, Y_{x_{1}}^{\vee}=\left\{x_{3}\right\} ; \overline{G R}_{B}(X)\left(x_{5}\right)=$ $\frac{3}{4}, Y_{x_{5}}^{\vee}=\left\{x_{6}\right\} ; \overline{G R}_{B}(X)\left(x_{7}\right)=\frac{3}{4}, Y_{x_{7}}^{\vee}=\left\{x_{6}\right\} ; \overline{G R}_{B}(X)\left(x_{j}\right)=$ $1, x_{j} \in\left\{x_{2}, x_{3}, x_{6}, x_{8}\right\}$.

From the Step 2 of Algorithm 4.4 we have a new relation matrix $M_{3 \times 3}^{*}$ :

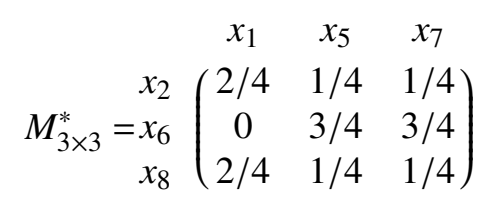

The lower and upper approximations of $X^{\wedge}=$ $\left\{x_{2}, x_{6}, x_{8}\right\}$ by removing $x_{3}$ from $X$ be updated as follows:

From the step 3 of algorithm 4.4 we have the lower approximations:

$\underline{G R}_{B}^{\wedge}\left(X^{\wedge}\right)\left(x_{i}\right)=\underline{G R}_{B}(X)\left(x_{i}\right), \quad Y_{x_{i}}^{\wedge *}=Y_{x_{i}}^{\wedge}, \quad x_{i} \in$ $\left\{x_{2}, x_{6}, x_{8}\right\}$, and $G R_{B}^{\wedge} \overline{\left(X^{\wedge}\right)}\left(x_{i}\right)=0, x_{i} \in\left\{x_{1}, x_{5}, x_{7}\right\}$.

From the step 4 of algorithm 4.4 we have the upper approximations:

$\overline{G R}_{B}^{\wedge}\left(X^{\wedge}\right)\left(x_{1}\right)$ : Since $\overline{G R}_{B}(X)\left(x_{1}\right)=G R_{B}\left(x_{1}, x_{3}\right)$, then $\overline{G R_{B}^{\wedge}}\left(X^{\wedge}\right)\left(x_{1}\right)=\underset{y \in\left\{x_{2}, x_{6}, x_{8}\right\}}{\vee} G R_{B}\left(x_{1}, y\right)=\frac{2}{4}$ and $Y_{x_{1}}^{\vee *}=\left\{y ; \underset{y \in\left\{x_{2}, x_{6}, x_{8}\right\}}{\vee} G R_{B}\left(x_{1}, y\right)\right\}=\left\{x_{2}, x_{8}\right\}$.

$\overline{G R}_{B}^{\wedge}\left(X^{\wedge}\right)\left(x_{5}\right)$ : Since $Y_{x_{5}}^{\vee}=\left\{x_{6}\right\}, \overline{G R}_{B}(X)\left(x_{5}\right)=$ $G R_{B}\left(x_{5}, x_{6}\right) \neq G R_{B}\left(x_{5}, x_{3}\right)$, then $\overline{G R}_{B}^{\wedge}\left(X^{\wedge}\right)\left(x_{5}\right)=$ $\overline{G R}_{B}(X)\left(x_{5}\right)=\frac{3}{4}$ and $Y_{x_{5}}^{\vee *}=Y_{x_{5}}^{\vee}-\left\{x_{3}\right\}=\left\{x_{6}\right\}$.

$\overline{G R}_{B}^{\wedge}\left(X^{\wedge}\right)\left(x_{7}\right)$ : Since $Y_{x_{7}}^{\vee}=\left\{x_{6}\right\}, \overline{G R}_{B}(X)\left(x_{7}\right)=$ $G R_{B}\left(x_{7}, x_{6}\right) \neq G R_{B}\left(x_{7}, x_{3}\right)$, then $\overline{G R}{ }_{B}^{\wedge}\left(X^{\wedge}\right)\left(x_{7}\right)=$ $\overline{G R}_{B}(X)\left(x_{7}\right)=\frac{3}{4}$ and $Y_{x_{7}}^{\vee *}=Y_{x_{7}}^{\vee}-\left\{x_{3}\right\}=\left\{x_{6}\right\}$.

$$
\overline{G R}_{B}^{\wedge}\left(X^{\wedge}\right)\left(x_{j}\right)=\overline{G R}_{B}(X)\left(x_{j}\right)=1, x_{j} \in\left\{x_{2}, x_{6}, x_{8}\right\} .
$$

\section{Incrementally updating approximations when changing the attribute value of the object}

In practical situation, the attribute values of the object are likely to change, as well. In this section, we discuss the methods of incrementally updating approximations based on the grade indiscernibility relation when changing the decision attribute value and the conditional attribute value of the object, respectively.

\subsection{Incrementally updating approximations when changing the decision attribute value of the object}

Proposition 11 Let $S=(U, A, V, F)$ be an information system, $B \subseteq A, X \subseteq U, x^{\vee} \in U$ and $x^{\vee} \notin X$. The lower and upper approximations of $X^{\vee}=X \cup\left\{x^{\vee}\right\}$ by changing the decision attribute value of $x^{\vee}$ from $U-X$ to $X$ can be updated respectively as follows.

Lower approximation: If $x_{i} \in U-X^{\vee}$, we have $\underline{G R}_{B}\left(X^{\vee}\right)\left(x_{i}\right)=\underline{G R}_{B}(X)\left(x_{i}\right)=0$. If $x_{i} \in X^{\vee}$, we have: If $\exists y \in Y_{x_{i}}^{\wedge}$ such that $\underline{G R}_{B}(X)\left(x_{i}\right)=1-$ $G R_{B}\left(x_{i}, y\right) \neq 1-G R_{B}\left(x_{i}, x^{\vee}\right)$, then $\underline{G R}_{B}\left(X^{\vee}\right)\left(x_{i}\right)=$ $\underline{G R}_{B}(X)\left(x_{i}\right)$ and $Y_{x_{i}}^{\wedge *}=Y_{x_{i}}^{\wedge}-\left\{x^{\vee}\right\}$.

If $G R_{B}(X)\left(x_{i}\right) \stackrel{x_{i}}{=} 1-G R_{B}\left(x_{i}, x^{\vee}\right)$ or $x_{i}=x^{\vee}$, then $\underline{G R}_{B}\left(\bar{X}^{\vee}\right)\left(x_{i}\right)=\wedge_{y \in U-X^{\vee}}^{\wedge}\left(1-G R_{B}\left(x_{i}, y\right)\right)$ and $Y_{x_{i}}^{\wedge *}=$ $\left\{y ; \underset{y \in U-X^{\vee}}{\vee} G R_{B}\left(x_{i}, y\right)\right\}$.

Upper approximation: If $x_{j} \in X^{\vee}$, we have $\overline{G R}_{B}\left(X^{\vee}\right)\left(x_{j}\right)=1$. If $x_{j} \in U-X^{\vee}$, we have:

If $G R_{B}\left(x_{j}, x^{\vee}\right)>\overline{G R}_{B}(X)\left(x_{j}\right)$, then $\overline{G R}_{B}\left(X^{\vee}\right)\left(x_{j}\right)=$ $G R_{B}\left(x_{j}, x^{\vee}\right)$ and $Y_{x_{j}}^{\vee *}=\left\{x^{\vee}\right\}$.

If $G R_{B}\left(x_{j}, x^{\vee}\right)=\overline{G R}_{B}(X)\left(x_{j}\right)$, then $\overline{G R}_{B}\left(X^{\vee}\right)\left(x_{j}\right)=$ $\overline{G R}_{B}(X)\left(x_{j}\right)$ and $Y_{x_{j}}^{\vee *}=Y_{x_{j}}^{\vee} \cup\left\{x^{\vee}\right\}$.

If $\operatorname{IR}_{B}\left(x_{j}, x^{\vee}\right)<\overline{G R}_{B}(X)\left(x_{j}\right)$, then $\overline{G R}_{B}\left(X^{\vee}\right)\left(x_{j}\right)=$ $\overline{G R}_{B}(X)\left(x_{j}\right)$ and $Y_{x_{j}}^{\vee *}=Y_{x_{j}}^{\vee}$.

Algorithm 5.1 (Incremental algorithm for updating approximations when changing the decision attribute value of $x^{\vee}, x^{\vee} \in U-X$ to $x^{\vee} \in X$ )

Step 1: Input the relation matrix $M_{n \times m}$; $\underline{G R}_{B}(X)\left(x_{i}\right), Y_{x_{i}}^{\wedge}, x_{i} \in X ; \overline{G R}_{B}(X)\left(x_{j}, Y_{x_{j}}^{\vee}, x_{j} \in U-X\right.$.

Step 2: We get a new relation matrix $M_{(n+1) \times(m-1)}^{*}$. // Delete $G R_{B}\left(x^{\vee}, x_{i}\right), x_{i} \in X$ from the relation matrix $M_{n \times m}$ and calculate $G R_{B}\left(x^{\vee}, x_{j}\right), x_{j} \in$ $U-X^{\wedge}$. $X^{\vee}$

Step 3: Calculate the lower approximations $x_{i} \in$

If $\exists y \in Y_{x_{i}}^{\wedge}$, such that $\underline{G R}_{B}(X)\left(x_{i}\right)=1-$ $G R_{B}\left(x_{i}, y\right) \neq 1-G R_{B}\left(x_{i}, x^{\vee}\right)$, then $\underline{G R}_{B}\left(X^{\vee}\right)\left(x_{i}\right)=$ $\underline{G R}_{B}(X)\left(x_{i}\right)$ and $Y_{x_{i}}^{\wedge *}=Y_{x_{i}}^{\wedge}-\left\{x^{\vee}\right\}$.

Else $\underline{G R}_{B}\left(X^{\vee}\right)\left(x_{i}\right)=\bigwedge_{y \in U-X^{\vee}}^{\wedge}\left(1-G R_{B}\left(x_{i}, y\right)\right)$ and $Y_{x_{i}}^{\wedge *}=\left\{y ; \underset{y \in U-X^{\vee}}{\vee} G R_{B}\left(x_{i}, y\right)\right\}$. // According to the Proposition 11.

Step 4: Calculate the upper approximations $x_{j} \in U-X^{\vee}$ 
If $G R_{B}\left(x_{j}, x^{\vee}\right)>\overline{G R}_{B}(X)\left(x_{j}\right)$, then $\overline{G R}_{B}\left(X^{\vee}\right)\left(x_{j}\right)=$ $G R_{B}\left(x_{j}, x^{\vee}\right)$ and $Y_{x_{j} *}^{\vee *}=\left\{x^{\vee}\right\}$.

If $G R_{B}\left(x_{j}, x^{\vee}\right)=\overline{G R}_{B}(X)\left(x_{j}\right)$, then $\overline{G R}_{B}\left(X^{\vee}\right)\left(x_{j}\right)=$ $\overline{G R}_{B}(X)\left(x_{j}\right)$ and $Y_{x_{j}}^{\vee *}=Y_{x_{j}}^{\vee} \cup\left\{x^{\vee}\right\}$.

If $G R_{B}\left(x_{j}, x^{\vee}\right)<\overline{G R}_{B}(X)\left(x_{j}\right)$, then $\overline{G R}_{B}\left(X^{\vee}\right)\left(x_{j}\right)=$ $\overline{G R}_{B}(X)\left(x_{j}\right)$ and $Y_{x_{j}}^{\vee *}=Y_{x_{j}}^{\vee}$. // According to the Proposition 11.

Step 5: Output the relation matrix $M_{(n+1) \times(m-1)}^{*}$; $\underline{G R}_{B}\left(X^{\vee}\right)\left(x_{i}\right), \quad Y_{x_{i}}^{\wedge *}, \quad x_{i} \in X^{\vee} ; \quad \overline{G R}_{B}\left(X^{\vee}\right)\left(x_{j}\right), \quad Y_{x_{j} *}^{\vee *}$, $x_{j} \in U-X^{\vee} ; \underline{G R}_{B}\left(X^{\vee}\right)\left(x_{i}\right)=0, x_{i} \in U-X^{\vee}$, and $\overline{G R}_{B}\left(X^{\vee}\right)\left(x_{j}\right)=1, x_{j} \in X^{\vee}$.

The Algorithm 5.1 has a time complexity of $O(|U-X||B|)$, which is mainly decided by Step 2 . In the following Example 8, We use the results from Example 7 to demonstrate how algorithm 5.1 update the approximations when changing the decision value of the object from $U-X$ to $X$.

Example 8 We consider the information system given in Table 6. Let $U=\left\{x_{1}, x_{2}, x_{5}, x_{6}, x_{7}, x_{8}\right\}$ be the universal set, $B=\left\{b_{1}, b_{3}, b_{4}, b_{5}\right\}$ be the conditional attribute set, $X=\left\{x_{2}, x_{6}, x_{8}\right\}$ be the decision set. Changing the decision attribute value of $x_{5}$ to $X$ from $U-X, X^{\vee}=X \cup\left\{x_{5}\right\}$.

\begin{tabular}{cccccc}
\multicolumn{6}{c}{ Table 7.} \\
\hline & $b_{1}$ & $b_{3}$ & $b_{4}$ & $b_{5}$ & $d$ \\
\hline$x_{1}$ & 2 & 1 & 1 & 3 & $U-X$ \\
$x_{2}$ & 2 & 1 & 2 & 2 & $X$ \\
$x_{5}$ & 1 & 2 & 2 & 3 & $X$ \\
$x_{6}$ & 1 & 2 & 2 & 2 & $X$ \\
$x_{7}$ & 1 & 2 & 2 & 1 & $U-X$ \\
$x_{8}$ & 2 & 1 & 2 & 2 & $X$ \\
\hline
\end{tabular}

Using the result of Example 7.

$$
\begin{array}{rccc}
x_{1} & x_{5} & x_{7} \\
M_{3 \times 3}=x_{2} & x_{6} \\
x_{8}
\end{array}\left(\begin{array}{ccc}
2 / 4 & 1 / 4 & 1 / 4 \\
0 & 3 / 4 & 3 / 4 \\
2 / 4 & 1 / 4 & 1 / 4
\end{array}\right)
$$

$\underline{G R}_{B}(X)\left(x_{2}\right)=\frac{2}{4}, Y_{x_{2}}^{\wedge}=\left\{x_{1}\right\} ; \underline{G R}_{B}(X)\left(x_{6}\right)=\frac{1}{4}, Y_{x_{6}}^{\wedge}=$ $\left\{x_{5}, x_{7}\right\} ; \underline{G R}_{B}(X)\left(x_{8}\right)=\frac{2}{4}, Y_{x_{8}}^{\wedge}=\left\{x_{1}\right\} ; \underline{G R}_{B}(X)\left(x_{i}\right)=$ $0, x_{i} \in\left\{x_{1}, x_{5}, x_{7}\right\}$.

$\overline{G R}_{B}(X)\left(x_{1}\right)=\frac{2}{4}, Y_{x_{1}}^{\vee}=\left\{x_{2}, x_{8}\right\} ; \overline{G R}_{B}(X)\left(x_{5}\right)=$ $\frac{3}{4}, Y_{x_{5}}^{\vee}=\left\{x_{6}\right\} ; \overline{G R}_{B}(X)\left(x_{7}\right)=\frac{3}{4}, Y_{x_{7}}^{\vee}=\left\{x_{6}\right\} ; \overline{G R}_{B}(X)\left(x_{j}\right)=$ $1, x_{j} \in\left\{x_{2}, x_{6}, x_{8}\right\}$.
From the Step 2 of Algorithm 5.1 we have a new relation matrix $M_{4 \times 2}^{*}$ :

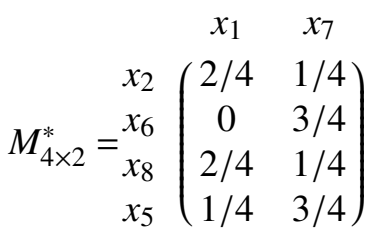

The lower and upper approximations of $X^{\vee}=$ $\left\{x_{2}, x_{5}, x_{6}, x_{8}\right\}$ by changing the decision attribute value $x_{5} \in U-X$ to $x_{5} \in X$ are updated as follows:

From the step 3 of algorithm 5.1 we have the lower approximations:

$\underline{G R}_{B}\left(X^{\vee}\right)\left(x_{2}\right)$ : Since $Y_{x_{2}}^{\wedge}=\left\{x_{1}\right\}, \underline{G R}_{B}(X)\left(x_{2}\right)=$ $1-\overline{G R}_{B}\left(x_{2}, x_{1}\right)$, then $\underline{G R}_{B}\left(X^{\vee}\right)\left(x_{2}\right)=\underline{G R}_{B}(X)\left(x_{2}\right)=$ $\frac{2}{4}$ and $Y_{x_{2}}^{\wedge *}=Y_{x_{2}}^{\wedge}-\left\{x_{5}\right\}=\left\{x_{1}\right\}$.

$\underline{G R}_{B}\left(X^{\vee}\right)\left(x_{6}\right):$ Since $\exists x_{7} \in Y_{x_{6}}^{\wedge}$, such that $\underline{G R}_{B}(X)\left(x_{6}\right)=1-G R_{B}\left(x_{6}, x_{7}\right)$, then $\underline{G R}_{B}\left(X^{\vee}\right)\left(x_{6}\right)=$ $\underline{G R}_{B}(X)\left(x_{6}\right)=\frac{1}{4}$ and $Y_{x_{6}}^{\wedge *}=Y_{x_{6}}^{\wedge}-\left\{x_{5}\right\}=\left\{x_{7}\right\}$.

$\underline{G R}_{B}\left(X^{\vee}\right)\left(x_{8}\right)$ : Since $Y_{x_{8}}^{\wedge}=\left\{x_{1}\right\}, \underline{G R}_{B}(X)\left(x_{8}\right)=$ $1-\overline{G R}_{B}\left(x_{8}, x_{1}\right)$, then $\underline{G R}_{B}\left(X^{\vee}\right)\left(x_{8}\right)=\underline{G R}_{B}(X)\left(x_{8}\right)=$ $\frac{2}{4}$ and $Y_{x_{8}}^{\wedge *}=Y_{x_{8}}^{\wedge}-\left\{x_{5}\right\}=\left\{x_{1}\right\}$.

$$
\underline{G R}_{B}\left(X^{\vee}\right)\left(x_{5}\right): \quad \underline{G R}_{B}\left(X^{\vee}\right)\left(x_{5}\right)=\wedge_{y \in\left\{x_{1}, x_{7}\right\}}^{\wedge}(1-
$$
$\left.G R_{B}\left(x_{5}, y\right)\right)=\frac{1}{4}$ and $Y_{x_{8}}^{\wedge *}=\left\{y ; \underset{y \in\left\{x_{1}, x_{7}\right\}}{\vee} G R_{B}\left(x_{5}, y\right)\right\}=$ $\left\{x_{7}\right\}$.

$\underline{G R}_{B}\left(X^{\vee}\right)\left(x_{i}\right)=\underline{G R}_{B}(X)\left(x_{i}\right)=0, x \in\left\{x_{1}, x_{7}\right\}$.

From the step 4 of algorithm 5.1 we have the upper approximations:

$\overline{G R}_{B}\left(X^{\vee}\right)\left(x_{1}\right)$ : Since $G R_{B}\left(x_{1}, x_{5}\right)<\overline{G R}_{B}(X)\left(x_{1}\right)$, then $\overline{G R}_{B}\left(X^{\vee}\right)\left(x_{1}\right)=\overline{G R}_{B}(X)\left(x_{1}\right)=\frac{2}{4}$ and $Y_{x_{1}}^{\vee *}=$ $Y_{x_{1}}^{\vee}=\left\{x_{2}, x_{8}\right\}$.

$\overline{G R}_{B}\left(X^{\vee}\right)\left(x_{7}\right)$ : Since $G R_{B}\left(x_{7}, x_{5}\right)=\overline{G R}_{B}(X)\left(x_{7}\right)$, then $\overline{G R}_{B}\left(X^{\vee}\right)\left(x_{7}\right)=\overline{G R}_{B}(X)\left(x_{7}\right)=\frac{3}{4}$ and $Y_{x_{7}}^{\vee *}=$ $Y_{x_{7}}^{\vee} \cup\left\{x_{5}\right\}=\left\{x_{5}, x_{6}\right\}$.

$\overline{G R}_{B}\left(X^{\vee}\right)\left(x_{j}\right)=\overline{G R}_{B}(X)\left(x_{j}\right)=1, x_{j} \in$ $\left\{x_{2}, x_{5}, x_{6}, x_{8}\right\}$.

Proposition 12 Let $S=(U, A, V, F)$ be an information system, $B \subseteq A, X \subseteq U, x^{\wedge} \in X$. The lower and upper approximations of $X^{\wedge}=X-\left\{x^{\wedge}\right\}$ by changing the decision attribute value of $x^{\wedge}$ from $X$ to $U-X$ can be updated respectively as follows.

Lower approximation: If $x_{i} \in U-X^{\wedge}$, we have $\underline{G R}_{B}\left(X^{\wedge}\right)\left(x_{i}\right)=0$; If $x_{i} \in X^{\wedge}$, we have: 
If $1-G R_{B}\left(x_{i}, x^{\wedge}\right)>G R_{B}(X)\left(x_{i}\right)$, then $\underline{G R}_{B}\left(X^{\wedge}\right)\left(x_{i}\right)=\underline{G R}_{B}(X)\left(x_{i}\right)$ and $Y_{x_{i}}^{\wedge *}=Y_{x_{i}}^{\wedge}$

If $1-G R_{B}\left(x_{i}, x^{\wedge}\right)=G R_{B}(X)\left(x_{i}\right)$, then $\underline{G R}_{B}\left(X^{\wedge}\right)\left(x_{i}\right)=\underline{G R}_{B}(X)\left(x_{i}\right)$ and $Y_{x_{i}}^{\wedge}=Y_{x_{i}}^{\wedge} \cup\left\{x^{\wedge}\right\}$.

If $1-G R_{B}\left(x_{i}, x^{\wedge}\right)<\underline{G R}_{B}(X)\left(x_{i}\right)$, then $\underline{G R}_{B}\left(X^{\wedge}\right)\left(x_{i}\right)=1-G R_{B}\left(x_{i}, x^{\wedge}\right)$ and $Y_{x_{i}}^{\wedge *}=\left\{x^{\wedge}\right\}$.

Upper approximation: If $x_{j} \in X^{\wedge}$, we have $\overline{G R}_{B}\left(X^{\wedge}\right)\left(x_{j}\right)=\overline{G R}_{B}(X)\left(x_{j}\right)=1$; If $x_{j} \in U-X^{\wedge}$, we have:

If $\exists y \in Y_{x_{j}}^{\vee}$, such that $\overline{G R}_{B}(X)\left(x_{j}\right)=G R_{B}\left(x_{j}, y\right)$, then $\overline{G R}_{B}\left(X^{\wedge}\right)\left(x_{j}\right)=\overline{G R}_{B}(X)\left(x_{j}\right)$ and $Y_{x_{j}}^{\vee *}=Y_{x_{j}}^{\vee}-$ $\left\{x^{\wedge}\right\}$.

If $\overline{G R}_{B}(X)\left(x_{j}\right)=G R_{B}\left(x_{j}, x^{\wedge}\right)$, or $x_{j}=x^{\wedge}$, then $\overline{G R}_{B}(X)\left(x_{j}\right)=\underset{y \in X^{\wedge}}{\vee} G R_{B}\left(x_{j}, y\right)$ and $Y_{x_{j}}^{\vee *}=$ $\left\{y ; \underset{y \in X^{\wedge}}{\vee} G R_{B}\left(x_{j}, y\right)\right\}$.

Algorithm 5.2 (Incremental algorithm for updating approximations when changing the decision attribute value of $x^{\wedge}, x^{\wedge} \in X$ to $x^{\wedge} \in U-X$ )

Step 1: Input the relation matrix $M_{n \times m}$; $\underline{G R}_{B}(X)\left(x_{i}\right), Y_{x_{i}}^{\wedge}, x_{i} \in X ; \overline{G R}_{B}(X)\left(x_{j}, Y_{x_{j}}^{\vee}, x_{j} \in U-X\right.$.

Step 2: We get a new relation matrix $M_{(n+1) \times(m-1)}^{*} \quad \quad / /$ Delete $G R_{B}\left(x^{\wedge}, x_{j}\right), x_{j} \in U-$ $X$ from the relation matrix $M_{n \times m}$ and calculate $G R_{B}\left(x^{\vee}, x_{i}\right), x_{i} \in X^{\vee}$.

Step 3: Calculate the lower approximations $x_{i} \in$ $X^{\wedge}$

If $1-G R_{B}\left(x_{i}, x^{\wedge}\right)>G R_{B}(X)\left(x_{i}\right)$, then $\underline{G R}_{B}\left(X^{\wedge}\right)\left(x_{i}\right)=\underline{G R}_{B}(X)\left(x_{i}\right)$ and $\bar{Y}_{x_{i}}^{\wedge}=Y_{x_{i}}^{\wedge}$;

If $1-G R_{B}\left(x_{i}, x^{\wedge}\right)=G R_{B}(X)\left(x_{i}\right)$, then $\underline{G R}_{B}\left(X^{\wedge}\right)\left(x_{i}\right)=\underline{G R}_{B}(X)\left(x_{i}\right)$ and $Y_{x_{i}}^{\wedge *}=Y_{x_{i}}^{\wedge} \cup\left\{x^{\wedge}\right\}$;

If $1-G R_{B}\left(x_{i}, x^{\wedge}\right)<G R_{B}(X)\left(x_{i}\right)$, then $\underline{G R}_{B}\left(X^{\wedge}\right)\left(x_{i}\right)=1-G R_{B}\left(x_{i}, x^{\wedge}\right)$ and $Y_{x_{i}}^{\wedge *}=\left\{x^{\wedge}\right\} . / /$ According to the Proposition 12.

Step 4: Calculate the upper approximations $x_{j} \in$ $U-X^{\wedge}$

If $\exists y \in Y_{x_{j}}^{\vee}$, such that $\overline{G R}_{B}(X)\left(x_{j}\right)=G R_{B}\left(x_{j}, y\right)$, then $\overline{G R}_{B}\left(X^{\wedge}\right)\left(x_{j}\right)=\overline{G R}_{B}(X)\left(x_{j}\right)$ and $Y_{x_{j}}^{\vee *}=Y_{x_{j}}^{\vee}-$ $\left\{x^{\wedge}\right\}$;

Else $\overline{G R}_{B}(X)\left(x_{j}\right)=\underset{y \in X^{\wedge}}{\vee} G R_{B}\left(x_{j}, y\right)$ and $Y_{x_{j}}^{\vee *}=$ $\left\{y ; \underset{y \in X^{\wedge}}{\vee} G R_{B}\left(x_{j}, y\right)\right\}$. // According to the Proposition 12.

Step 5: Output the relation matrix $M_{(n+1) \times(m-1)}^{*}$; $\underline{G R}_{B}\left(X^{\wedge}\right)\left(x_{i}\right), Y_{x_{i}}^{\wedge *}, x_{i} \in X^{\wedge} ; \overline{G R}_{B}\left(X^{\wedge}\right)\left(x_{j}\right)$ and $Y_{x_{j}}^{\vee *}$, $x_{j} \in U-X^{\wedge} ; \underline{G R}_{B}\left(X^{\wedge}\right)\left(x_{i}\right)=0, x_{i} \in U-X^{\wedge}$, and $\overline{G R}_{B}\left(X^{\wedge}\right)\left(x_{j}\right)=1, x_{j} \in X^{\wedge}$.

The Algorithm 5.2 has a time complexity of $O(|X||B|)$, which is mainly decided by Step 2 . In the following Example 9, We use the results from Example 8 to demonstrate how algorithm 5.2 update the approximations when changing the decision attribute value of the object from $X$ to $U-X$.

Example 9 We consider the information system given in Table 7. Let $U=\left\{x_{1}, x_{2}, x_{5}, x_{6}, x_{7}, x_{8}\right\}$ be the universal set, $B=\left\{b_{1}, b_{3}, b_{4}, b_{5}\right\}$ be the conditional attribute set, $X=\left\{x_{2}, x_{5}, x_{6}, x_{8}\right\}$ be the decision set. Changing the decision attribute value of $x_{8}$ from $X$ to $U-X, X^{\wedge}=\left\{x_{2}, x_{5}, x_{6}\right\}$.

\begin{tabular}{cccccc}
\multicolumn{6}{c}{ Table 8.} \\
\hline & $b_{1}$ & $b_{3}$ & $b_{4}$ & $b_{5}$ & $d$ \\
\hline$x_{1}$ & 2 & 1 & 1 & 3 & $U-X$ \\
$x_{2}$ & 2 & 1 & 2 & 2 & $X$ \\
$x_{5}$ & 1 & 2 & 2 & 3 & $X$ \\
$x_{6}$ & 1 & 2 & 2 & 2 & $X$ \\
$x_{7}$ & 1 & 2 & 2 & 1 & $U-X$ \\
$x_{8}$ & 2 & 1 & 2 & 2 & $U-X$ \\
\hline
\end{tabular}

Using the result of Example 8.

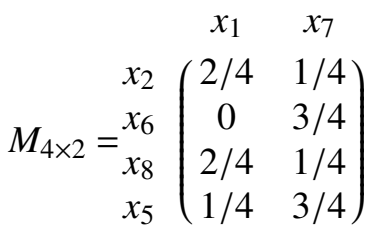

$\underline{G R}_{B}(X)\left(x_{2}\right)=\frac{2}{4}, Y_{x_{2}}^{\wedge}=\left\{x_{1}\right\} ; \underline{G R}_{B}(X)\left(x_{6}\right)=\frac{1}{4}, Y_{x_{6}}^{\wedge}=$ $\left\{x_{7}\right\} ; \quad \underline{G R}_{B}(X)\left(x_{8}\right)=\frac{2}{4}, Y_{x_{8}}^{\wedge}=\left\{x_{1}\right\} ; \quad \underline{G R}_{B}(X)\left(x_{5}\right)=$ $\frac{1}{4}, Y_{x_{5}}^{\wedge}=\left\{x_{7}\right\} ; \underline{G R}_{B}(X)\left(x_{i}\right)=0, x_{i} \in\left\{x_{1}, x_{7}\right\}$.

$\overline{G R}_{B}(X)\left(x_{1}\right)=\frac{2}{4}, Y_{x_{1}}^{\vee}=\left\{x_{2}, x_{8}\right\} ; \overline{G R}_{B}(X)\left(x_{7}\right)=$ $\frac{3}{4}, Y_{x_{7}}^{\vee}=\left\{x_{5}, x_{6}\right\} ; \overline{G R}_{B}(X)\left(x_{j}\right)=1, x_{j} \in\left\{x_{2}, x_{5}, x_{6}, x_{8}\right\}$.

From the Step 2 of Algorithm 5.2 we have a new relation matrix $M_{3 \times 3}^{*}$ :

$$
\begin{array}{r}
x_{1 \times 3} \\
M_{3}^{*} x_{6} \\
x_{5}
\end{array}\left(\begin{array}{ccc}
2 / 4 & x_{7} & x_{8} \\
0 & 3 / 4 & 4 / 4 \\
1 / 4 & 3 / 4 & 2 / 4 \\
1 / 4
\end{array}\right)
$$

The lower and upper approximations of $X^{\wedge}=$ $\left\{x_{2}, x_{5}, x_{6}\right\}$ by changing the decision attribute value $x_{8} \in X$ to $x_{8} \in U-X$,are updated as follows: 
From the step 3 of algorithm 5.2 we have the lower approximations:

$$
\underline{G R}_{B}\left(X^{\wedge}\right)\left(x_{2}\right): \quad \text { Since } 1-G R_{B}\left(x_{2}, x_{8}\right)<
$$
$\underline{G R}_{B}(X)\left(x_{2}\right)$, then $\underline{G R}_{B}\left(X^{\wedge}\right)\left(x_{2}\right)=1-G R_{B}\left(x_{2}, x_{8}\right)=$ 0 and $Y_{x_{2}}^{\wedge *}=\left\{x_{8}\right\}$.

$\underline{G R}_{B}\left(X^{\wedge}\right)\left(x_{6}\right): \quad$ Since $1-G R_{B}\left(x_{6}, x_{8}\right)>$ $\underline{G R}_{B}(X)\left(x_{6}\right)$, then $\underline{G R}_{B}\left(X^{\wedge}\right)\left(x_{6}\right)=\underline{G R}_{B}(X)\left(x_{6}\right)=\frac{1}{4}$ and $Y_{x_{6}}^{\wedge *}=Y_{x_{6}}^{\wedge}=\left\{x_{7}\right\}$.

$\underline{G R}_{B}\left(X^{\wedge}\right)\left(x_{5}\right): \quad$ Since $1-G R_{B}\left(x_{5}, x_{8}\right)>$ $\underline{G R}_{B}(X)\left(x_{5}\right)$, then $\underline{G R}_{B}\left(X^{\wedge}\right)\left(x_{5}\right)=\underline{G R}_{B}(X)\left(x_{5}\right)=\frac{1}{4}$ and $Y_{x_{5}}^{\wedge *}=Y_{x_{5}}^{\wedge}=\left\{x_{7}\right\}$.

$\underline{G R}_{B}\left(X^{\wedge}\right)\left(x_{i}\right)=\underline{G R}_{B}(X)\left(x_{i}\right)=0, x \in\left\{x_{1}, x_{7}, x_{8}\right\}$.

From the step 4 of algorithm 5.2 we have the upper approximations:

$\overline{G R}_{B}\left(X^{\wedge}\right)\left(x_{1}\right):$ Since $\exists x_{2} \in Y_{x_{1}}^{\vee}$, such that $\overline{G R}_{B}(X)\left(x_{1}\right)=G R_{B}\left(x_{1}, x_{2}\right)$, then $\overline{G R}_{B}\left(X^{\wedge}\right)\left(x_{1}\right)=$ $\overline{G R}_{B}(X)\left(x_{1}\right)=\frac{2}{4}$ and $Y_{x_{1}}^{\vee *}=Y_{x_{1}}^{\vee}-\left\{x_{8}\right\}=\left\{x_{2}\right\}$.

$\overline{G R}_{B}\left(X^{\wedge}\right)\left(x_{7}\right):$ Since $\exists x_{6} \in Y_{x_{7}}^{\vee}$, such that $\overline{G R}_{B}(X)\left(x_{7}\right)=G R_{B}\left(x_{7}, x_{6}\right)$, then $\overline{G R}_{B}\left(X^{\wedge}\right)\left(x_{7}\right)=$ $\overline{G R}_{B}(X)\left(x_{7}\right)=\frac{3}{4}$ and $Y_{x_{7}}^{\vee *}=Y_{x_{7}}^{\vee}-\left\{x_{8}\right\}=\left\{x_{5}, x_{6}\right\}$.

$$
\overline{G R}_{B}\left(X^{\wedge}\right)\left(x_{8}\right): \overline{G R}_{B}(X)\left(x_{8}\right)=\underset{y \in\left\{x_{2}, x_{5}, x_{6}\right\}}{\vee} G R_{B}\left(x_{8}, y\right)=
$$
$\frac{4}{4}$ and $Y_{x_{8}}^{\vee *}=\left\{y ; \underset{y \in\left\{x_{2}, x_{5}, x_{6}\right\}}{\vee} G R_{B}\left(x_{8}, y\right)\right\}=\left\{x_{2}\right\}$.

$$
\overline{G R}_{B}\left(X^{\wedge}\right)\left(x_{j}\right)=\overline{G R}_{B}(X)\left(x_{j}\right)=1, x_{j} \in\left\{x_{2}, x_{5}, x_{6}\right\} .
$$

\subsection{Incremental updating approximations when changing the conditional attribute value of the object}

Proposition 13 Let $S=(U, A, V, F)$ be an information system, $B \subseteq A, b \in B$, changing the conditional attribute value $f\left(x^{*}, b\right)$ to $f^{*}\left(x^{*}, b\right), f^{*}\left(x^{*}, b\right) \neq$ $f\left(x^{*}, b\right), x^{*} \in U$. For any $y \in U$,

$$
G R_{B}^{*}\left(x^{*}, y\right)= \begin{cases}\frac{|B| \cdot G R_{B}\left(x^{*}, y\right)+1}{|B|}, & f^{*}\left(x^{*}, b\right)=f(y, b) ; \\ \frac{|B| \cdot G R_{B}\left(x^{*}, y\right)-1}{|B|}, & f^{*}\left(x^{*}, b\right) \neq f(y, b) .\end{cases}
$$

Proposition 14 Let $S=(U, A, V, F)$ be an information system, $B \subseteq A, X \subseteq U, x^{*} \in X$. The lower and upper approximations of $X$ by changing the conditional attribute value of $x^{*}, f\left(x^{*}, b\right)$ to $f^{*}\left(x^{*}, b\right)$ $\left(f\left(x^{*}, b\right) \neq f^{*}\left(x^{*}, b\right)\right)$ can be updated respectively as follows.

Lower approximation: If $x_{i} \in U-X$, then $\underline{G R}_{B}^{*}(X)\left(x_{i}\right)=0$. If $x_{i} \in X$, we have
If $x_{i} \in X-\left\{x^{*}\right\}$, then $G R_{B}^{*}(X)\left(x_{i}\right)=G R_{B}(X)\left(x_{i}\right)$ and $Y_{x_{i}}^{\wedge *}=Y_{x_{i}}^{\wedge}$

If $x_{i}=x^{*}$, then $\underline{G R}_{B}^{*}(X)\left(x^{*}\right)=\bigwedge_{y \in U-X}(1-$ $\left.G R_{B}^{*}\left(x^{*}, y\right)\right)$ and $Y_{x_{i}}^{\wedge *}=\left\{y ; \underset{y \in U-X}{\vee} G R_{B}^{*}\left(x^{*}, y\right)\right\}$.

Upper approximation: If $x_{j} \in X$, then $\overline{G R}_{B}^{*}(X)\left(x_{j}\right)=1$. If $x_{j} \in U-X$, we have:

If $\overline{G R}_{B}(X)\left(x_{j}\right)=G R_{B}\left(x_{j}, x^{*}\right)$, then $\overline{G R}_{B}^{*}(X)\left(x_{j}\right)=$ $\underset{y \in X}{\vee} G R_{B}^{*}\left(x_{j}, y\right)$ and $Y_{x_{j}}^{\vee *}=\left\{y ; \underset{y \in X}{\vee} G R_{B}^{*}\left(x_{j}, y\right)\right\}$.

If $\exists y \in Y_{x_{j}}^{\vee}$, such that $\overline{G R}_{B}(X)\left(x_{j}\right)=G R_{B}\left(x_{j}, y\right)$, we have:

If $\overline{G R}_{B}(X)\left(x_{j}\right)>G R_{B}^{*}\left(x_{j}, x^{*}\right)$, then $\overline{G R}_{B}^{*}(X)\left(x_{j}\right)=$ $\overline{G R}_{B}(X)\left(x_{j}\right)$ and $Y_{x_{j}}^{\vee *}=Y_{x_{j}}^{\vee}-\left\{x^{*}\right\}$.

If $\overline{G R}_{B}(X)\left(x_{j}\right)=G R_{B}^{*}\left(x_{j}, x^{*}\right)$, then $\overline{G R}_{B}^{*}(X)\left(x_{j}\right)=$ $\overline{G R}_{B}(X)\left(x_{j}\right)$ and $Y_{x_{j}}^{\vee *}=Y_{x_{j}}^{\vee} \cup\left\{x^{*}\right\}$.

If $\overline{G R}_{B}(X)\left(x_{j}\right)<G R_{B}^{*}\left(x_{j}, x^{*}\right)$, then $\overline{G R}_{B}^{*}(X)\left(x_{j}\right)=$ $G R_{B}^{*}\left(x_{j}, x^{*}\right)$ and $Y_{x_{j}}^{\vee *}=\left\{x^{*}\right\}$.

Algorithm 5.3 (Incremental algorithm for updating approximations when changing the conditional attribute value $f\left(x^{*}, b\right)$ to $\left.f^{*}\left(x^{*}, b\right), x^{*} \in X\right)$

Step 1: Input the relation matrix $M_{n \times m}$; $\underline{G R}_{B}(X)\left(x_{i}\right), Y_{x_{i}}^{\wedge}, x_{i} \in X ; \overline{G R}_{B}(X)\left(x_{j}\right), Y_{x_{i}}^{\vee}, x_{j} \in U-X$; The changing attribute value of $x^{*}: f\left(x^{*}, b\right)$.

Step 2: We get a new relation matrix $M_{n \times m}^{*}$. Changing the value $G R_{B}\left(x^{*}, y\right)$ to $G R_{B}^{*}\left(x^{*}, y\right)$ of the relation matrix $M_{n \times m}, G R_{B}^{*}\left(x^{*}, y\right)=$ $\left\{\begin{array}{ll}\frac{|B| \cdot G R_{B}\left(x^{*}, y\right)+1}{|B|}, & f^{*}\left(x^{*}, b\right)=f(y, b) ; \\ \frac{|B| \cdot G R_{B}\left(x^{*}, y\right)-1}{|B|}, & f^{*}\left(x^{*}, b\right) \neq f(y, b) .\end{array} \quad y \in U-X / /\right.$ According to Proposition 13. $X$

Step 3: Calculate the lower approximations $x_{i} \in$

If $x_{i} \in X-\left\{x^{*}\right\}$, then $\underline{G R}_{B}^{*}(X)\left(x_{i}\right)=\underline{G R} \underline{B}_{B}(X)\left(x_{i}\right)$ and $Y_{x_{i}}^{\wedge *}=Y_{x_{i}}^{\wedge}$.

If $x_{i}=x^{*}$, then $\underline{G R}_{B}^{*}(X)\left(x^{*}\right)=\hat{y \in U}_{-X}^{\wedge}(1-$ $\left.G R_{B}^{*}\left(x^{*}, y\right)\right)$ and $Y_{x^{*}}^{\wedge *}=\left\{y ; \underset{y \in U-X}{\vee} G R_{B}^{*}\left(x^{*}, y\right)\right\}$. // According to Proposition 14.

Step 4: Calculate the upper approximations $x_{j} \in$ $U-X$

If $\exists y \in Y_{x_{j}}^{\vee}$, such that $\overline{G R}_{B}(X)\left(x_{j}\right)=G R_{B}\left(x_{j}, y\right)$, we have:

If $\overline{G R}_{B}(X)\left(x_{j}\right)>G R_{B}^{*}\left(x_{j}, x^{*}\right)$, then $\overline{G R}_{B}^{*}(X)\left(x_{j}\right)=$ $\overline{G R}_{B}(X)\left(x_{j}\right)$ and $Y_{x_{j}}^{\vee *}=Y_{x_{j}}^{\vee}-\left\{x^{*}\right\}$. 
If $\overline{G R}_{B}(X)\left(x_{j}\right)=G R_{B}^{*}\left(x_{j}, x^{*}\right)$, then $\overline{G R}_{B}^{*}(X)\left(x_{j}\right)=$ $\overline{G R}_{B}(X)\left(x_{j}\right)$ and $Y_{x_{j}}^{\vee *}=Y_{x_{j}}^{\vee} \cup\left\{x^{*}\right\}$.

If $\overline{G R}_{B}(X)\left(x_{j}\right)<G R_{B}^{*}\left(x_{j}, x^{*}\right)$, then $\overline{G R}_{B}^{*}(X)\left(x_{j}\right)=$ $G R_{B}^{*}\left(x_{j}, x^{*}\right)$ and $Y_{x_{j}}^{\vee *}=\left\{x^{*}\right\}$.

Else $\overline{G R}_{B}^{*}(X)\left(x_{j}\right)=\underset{y \in X}{\vee} G R_{B}^{*}\left(x_{j}, y\right)$ and $Y_{x_{j}}^{\vee *}=$ $\left\{y ; \underset{y \in X}{\vee} G R_{B}^{*}\left(x_{j}, y\right)\right\}$. // According to Proposition 14.

Step 5: Output the relation matrix $M_{n \times m}^{*}$; $\underline{G R}_{B}^{*}(X)\left(x_{i}\right), Y_{x_{i}}^{\wedge *}, x_{i} \in X ; \overline{G R}_{B}^{*}(X)\left(x_{j}\right), Y_{x_{j}}^{\vee *}, x_{j} \in U-$ $X ; \underline{G R}_{B}^{*}(X)\left(x_{i}\right)=0, x_{i} \in U-X$, and $\overline{G R}_{B}^{*}(X)\left(x_{j}\right)=1$, $x_{j} \in X$.

The Algorithm 5.3 has a time complexity of $O(|U-X||B|)$, which is mainly decided by Step 2 . In the following Example 10, We use the results from Example 9 to demonstrate how algorithm 5.3 update the approximations when changing the attribute value $f\left(x^{*}, b\right)$ to $f^{*}\left(x^{*}, b\right), x^{*} \in X$.

Example 10 We consider the information system given in Table 8. Let $U=\left\{x_{1}, x_{2}, x_{5}, x_{6}, x_{7}, x_{8}\right\}$ be the universal set, $B=\left\{b_{1}, b_{3}, b_{4}, b_{5}\right\}$ be the conditional attribute set, $X=\left\{x_{2}, x_{5}, x_{6}\right\}$ be the decision set. Changing $f\left(x_{5}, b_{3}\right)=2$ to $f^{*}\left(x_{5}, b_{3}\right)=1$.

\begin{tabular}{cccccc}
\multicolumn{6}{c}{ Table 9.} \\
\hline & $b_{1}$ & $b_{3}$ & $b_{4}$ & $b_{5}$ & $d$ \\
\hline$x_{1}$ & 2 & 1 & 1 & 3 & $U-X$ \\
$x_{2}$ & 2 & 1 & 2 & 2 & $X$ \\
$x_{5}$ & 1 & 1 & 2 & 3 & $X$ \\
$x_{6}$ & 1 & 2 & 2 & 2 & $X$ \\
$x_{7}$ & 1 & 2 & 2 & 1 & $U-X$ \\
$x_{8}$ & 2 & 1 & 2 & 2 & $U-X$ \\
\hline
\end{tabular}

Using the result of Example 9.

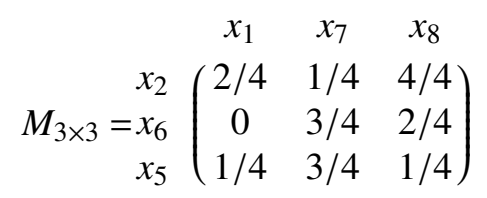

$\underline{G R}_{B}(X)\left(x_{2}\right)=0, Y_{x_{2}}^{\wedge}=\left\{x_{8}\right\} ; \underline{G R}_{B}(X)\left(x_{6}\right)=\frac{1}{4}, Y_{x_{6}}^{\wedge}=$ $\left\{x_{7}\right\} ; \quad \underline{G R}_{B}(X)\left(x_{5}\right)=\frac{1}{4}, Y_{x_{5}}^{\wedge}=\left\{x_{7}\right\} ; \quad \underline{G R}_{B}(X)\left(x_{i}\right)=$ $0, x_{i} \in\left\{x_{1}, x_{7}, x_{8}\right\}$.

$\overline{G R}_{B}(X)\left(x_{1}\right)=\frac{2}{4}, \quad Y_{x_{1}}^{\vee}=\left\{x_{2}\right\} ; \quad \overline{G R}_{B}(X)\left(x_{7}\right)=$ $\frac{3}{4}, \quad Y_{x_{7}}^{\vee}=\left\{x_{5}, x_{6}\right\} ; \quad \overline{G R}_{B}(X)\left(x_{8}\right)=\frac{4}{4}, \quad Y_{x_{8}}^{\vee}=\left\{x_{2}\right\}$; $\overline{G R}_{B}(X)\left(x_{j}\right)=1, x_{j} \in\left\{x_{2}, x_{5}, x_{6}\right\}$.
From the Step 2 of Algorithm 5.3 we have a new relation matrix $M_{3 \times 3}^{*}$ :

$$
\begin{array}{r}
x_{3 \times 3}=x_{2} \\
x_{2} \\
x_{5}
\end{array}\left(\begin{array}{ccc}
2 / 4 & 1 / 4 & 4 / 4 \\
0 & 3 / 4 & 2 / 4 \\
2 / 4 & 2 / 4 & 2 / 4
\end{array}\right)
$$

The lower and upper approximations of $X=$ $\left\{x_{2}, x_{5}, x_{6}\right\}$ by changing $f\left(x_{5}, b_{3}\right)$ to $f^{*}\left(x_{5}, b_{3}\right)$ are updated as follows.

From the step 3 of algorithm 5.3 we have the lower approximations:

$$
\begin{aligned}
& \quad \underline{G R}_{B}^{*}(X)\left(x_{i}\right)=G R_{B}(X)\left(x_{i}\right), Y_{x_{i}}^{\wedge *}=Y_{x_{i}}^{\wedge}, x_{i} \in\left\{x_{2}, x_{6}\right\} . \\
& \underline{G R}_{B}^{*}(X)\left(x_{5}\right)=\underset{y \in\left\{x_{1}, x_{7}, x_{8}\right\}}{\wedge}\left(1-G R_{B}^{*}\left(x_{5}, y\right)\right)=\frac{2}{4} \text { and } \\
& Y_{x_{5}}^{\wedge *}=\left\{y ; \underset{y \in\left\{x_{1}, x_{7}, x_{8}\right\}}{\vee} G R_{B}^{*}\left(x_{5}, y\right)\right\}=\left\{x_{1}, x_{7}, x_{8}\right\} .
\end{aligned}
$$

From the step 4 of algorithm 5.3 we have the upper approximations:

$\overline{G R}_{B}^{*}(X)\left(x_{1}\right):$ Since $\exists x_{2} \in Y_{x_{1}}^{\vee}, \overline{G R}_{B}(X)\left(x_{1}\right)=$ $G R_{B}\left(x_{1}, x_{2}\right)$, and $\overline{G R}_{B}(X)\left(x_{1}\right)=G R_{B}^{*}\left(x_{1}, x_{5}\right)$, then $\overline{G R}_{B}^{*}(X)\left(x_{1}\right)=\overline{G R}_{B}(X)\left(x_{1}\right)=\frac{2}{4}$ and $Y_{x_{1}}^{\vee *}=Y_{x_{1}}^{\vee} \cup$ $\left\{x_{5}\right\}=\left\{x_{2}, x_{5}\right\}$.

$\overline{G R}_{B}^{*}(X)\left(x_{7}\right):$ Since $\exists x_{6} \in Y_{x_{7}}^{\vee}, \overline{G R}_{B}(X)\left(x_{7}\right)=$ $G R_{B}\left(x_{7}, x_{6}\right)$, and $\overline{G R}_{B}(X)\left(x_{7}\right)>G R_{B}^{*}\left(x_{7}, x_{5}\right)$, then $\overline{G R}_{B}^{*}(X)\left(x_{7}\right)=\overline{G R}_{B}(X)\left(x_{7}\right)=\frac{3}{4}$, and $Y_{x_{7}}^{\vee *}=Y_{x_{7}}^{\vee}-$ $\left\{x_{5}\right\}=\left\{x_{6}\right\}$.

$\overline{G R}_{B}^{*}(X)\left(x_{8}\right):$ Since $\exists x_{2} \in Y_{x_{8}}^{\vee}, \overline{G R}_{B}(X)\left(x_{8}\right)=$ $G R_{B}\left(x_{8}, x_{2}\right)$, and $\overline{G R}_{B}(X)\left(x_{8}\right)>G R_{B}^{*}\left(x_{8}, x_{5}\right)$, then $\overline{G R}_{B}^{*}(X)\left(x_{8}\right)=\overline{G R}_{B}(X)\left(x_{8}\right)=\frac{4}{4}$, and $Y_{x_{8}}^{\vee *}=Y_{x_{8}}^{\vee}-$ $\left\{x_{5}\right\}=\left\{x_{2}\right\}$.

Proposition 15 Let $S=(U, A, V, F)$ be an information system, $B \subseteq A, X \subseteq U, x^{*} \in U-X$. The lower and upper approximations of $X$ by changing the conditional attribute value of $x^{*}, f\left(x^{*}, b\right)$ to $f^{*}\left(x^{*}, b\right)$ $\left(f\left(x^{*}, b\right) \neq f^{*}\left(x^{*}, b\right)\right)$ can be updated respectively as follows.

Lower approximation: If $x_{i} \in U-X$, then $\underline{G R}_{B}^{*}(X)\left(x_{i}\right)=0$. If $x_{i} \in X$, we have

If $\underline{G R} B(X)\left(x_{i}\right)=G R_{B}\left(x_{i}, x^{*}\right)$, then $G R_{B}^{*}(X)\left(x_{i}\right)=$

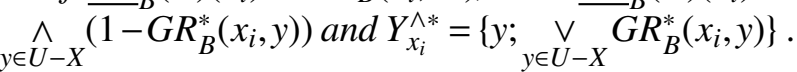
If $\exists y \in Y_{x_{i}}^{\wedge}$, such that $\underline{G R}_{B}(X)\left(x_{i}\right)=G R_{B}\left(x_{i}, y\right)$, we have:

If $\underline{G R}_{B}(X)\left(x_{i}\right)>G R_{B}^{*}\left(x_{i}, x^{*}\right)$, then $\underline{G R}_{B}^{*}(X)\left(x_{i}\right)=$ $\underline{G R}_{B}(X)\left(x_{i}\right)$ and $Y_{x_{i}}^{\wedge *}=Y_{x_{i}}^{\wedge}-\left\{x^{*}\right\}$; 
If $\underline{G R}_{B}(X)\left(x_{i}\right)=G R_{B}^{*}\left(x_{i}, x^{*}\right)$, then $\underline{G R}_{B}^{*}(X)\left(x_{i}\right)=$ $\underline{G R}_{B}(X)\left(x_{i}\right)$ and $Y_{x_{i}}^{\wedge *}=Y_{x_{i}}^{\wedge} \cup\left\{x^{*}\right\}$;

If $\underline{G R}_{B}(X)\left(x_{j}\right)<G R_{B}^{*}\left(x_{j}, x^{*}\right)$, then $\underline{G R}_{B}^{*}(X)\left(x_{j}\right)=$ $G R_{B}^{*}\left(x_{j}, x^{*}\right)$ and $Y_{x_{j}}^{\wedge *}=\left\{x^{*}\right\}$.

Upper approximation: If $x_{j} \in X$, then $\overline{G R}_{B}^{*}(X)\left(x_{j}\right)=1$. If $x_{j} \in U-X$, we have:

If $x_{j} \in U-X-\left\{x^{*}\right\}$, then $\overline{G R}_{B}^{*}(X)\left(x_{j}\right)=$ $\overline{G R}_{B}(X)\left(x_{j}\right)$ and $Y_{x_{j}}^{\vee *}=Y_{x_{j}}^{\vee}$.

If $x_{j}=x^{*}$, then $\overline{G R}_{B}^{*}(X)\left(x^{*}\right)=\underset{y \in X}{\vee} G R_{B}^{*}\left(x^{*}, y\right)$ and $Y_{x^{*}}^{\wedge *}=\left\{y ; \underset{y \in X}{\vee} G R_{B}^{*}\left(x^{*}, y\right)\right\}$.

Algorithm 5.4 (Incremental algorithm for updating approximations when changing the conditional attribute value $f\left(x^{*}, b\right)$ to $\left.f^{*}\left(x^{*}, b\right), x^{*} \in U-X\right)$

Step 1: Input the relation matrix $M_{n \times m}$; $\underline{G R}_{B}(X)\left(x_{i}\right), Y_{x_{i}}^{\wedge}, x_{i} \in X ; \overline{G R}_{B}(X)\left(x_{j}, Y_{x_{j}}^{\vee}, x_{j} \in U-X\right.$; The changing attribute value of $x^{*}: f\left(x^{*}, b\right)$.

Step 2: We get a new relation matrix $M_{n \times m}^{*}$. Changing the value $G R_{B}\left(x^{*}, y\right)$ to $G R_{B}^{*}\left(x^{*}, y\right)$ of the relation matrix $M_{n \times m}, G R_{B}^{*}\left(x^{*}, y\right)=$ $\left\{\begin{array}{ll}\frac{|B| \cdot G R_{B}\left(x^{*}, y\right)+1}{|B|}, & f^{*}\left(x^{*}, b\right)=f(y, b) ; \\ \frac{|B| \cdot G R_{B}\left(x^{*}, y\right)-1}{|B|}, & f^{*}\left(x^{*}, b\right) \neq f(y, b) .\end{array} \quad y \in X / /\right.$ According to Proposition 13. $X$

Step 3: Calculate the lower approximations $x_{i} \in$ have:

If $\exists y \in Y_{x_{i}}^{\wedge}$, such that $\underline{G R}_{B}(X)\left(x_{i}\right)=G R_{B}\left(x_{i}, y\right)$, we

If $\underline{G R}_{B}(X)\left(x_{i}\right)>G R_{B}^{*}\left(x_{i}, x^{*}\right)$, then $\underline{G R}_{B}^{*}(X)\left(x_{i}\right)=$ $\underline{G R}_{B}(X)\left(x_{i}\right)$ and $Y_{x_{i}}^{\wedge *}=Y_{x_{i}}^{\wedge}-\left\{x^{*}\right\}$;

If $\underline{G R_{B}}(X)\left(x_{i}\right)=G R_{B}^{*}\left(x_{i}, x^{*}\right)$, then $\underline{G R}_{B}^{*}(X)\left(x_{i}\right)=$

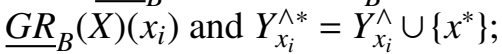

If $\underline{G R}_{B}(X)\left(x_{j}\right)<G R_{B}^{*}\left(x_{j}, x^{*}\right)$, then $\underline{G R}_{B}^{*}(X)\left(x_{j}\right)=$ $G R_{B}^{*}\left(x_{j}, x^{*}\right)$ and $Y_{x_{j}}^{\wedge *}=\left\{x^{*}\right\}$.

Else $\underline{G R}_{B}^{*}(X)\left(x_{i}\right)=\underset{y \in U-X}{\wedge}\left(1-G R_{B}^{*}\left(x_{i}, y\right)\right)$ and $Y_{x_{i}}^{\wedge *}=\left\{y ; \underset{y \in U-X}{\vee} G R_{B}^{*}\left(x_{i}, y\right)\right\}$. // According to Proposition 15. $U-X$

Step 4: Calculate the upper approximations $x_{j} \in$

If $x_{j} \in U-X-\left\{x^{*}\right\}$, then $\overline{G R}_{B}^{*}(X)\left(x_{j}\right)=$ $\overline{G R}_{B}(X)\left(x_{j}\right)$ and $Y_{x_{j}}^{\vee *}=Y_{x_{j}}^{\vee}$.

If $x_{j}=x^{*}$, then $\overline{G R}_{B}^{*}(X)\left(x^{*}\right)=\underset{y \in X}{\vee} G R_{B}^{*}\left(x^{*}, y\right)$ and $Y_{x^{*}}^{\wedge *}=\left\{y ; \underset{y \in X}{\vee} G R_{B}^{*}\left(x^{*}, y\right)\right\}$. // According to Proposition 15.
Step 5: Output the relation matrix $M_{n \times m}^{*}$; $\underline{G R}_{B}^{*}(X)\left(x_{i}\right), Y_{x_{i}}^{\wedge *}, x_{i} \in X ; \overline{G R}_{B}^{*}(X)\left(x_{j}\right), Y_{x_{j}}^{\vee *}, x_{j} \in U-$ $X ; \underline{G R}_{B}^{*}(X)\left(x_{i}\right)=0, x_{i} \in U-X$, and $\overline{G R}_{B}^{*}(X)\left(x_{j}\right)=1$, $x_{j} \in X$.

The time complexity of the Algorithm 5.4 is $O(|X||B|)$, which is mainly decided by the time cost of building the information system matrix in Step 2. In the following Example 11, We use the results from Example 10 to demonstrate how algorithm 5.4 update the approximations when changing the conditional attribute value $f\left(x^{*}, b\right)$ to $f^{*}\left(x^{*}, b\right)$, $x^{*} \in U-X$.

Example 11 We consider the information system given in Table 9. Let $U=\left\{x_{1}, x_{2}, x_{5}, x_{6}, x_{7}, x_{8}\right\}$ be the universal set, $B=\left\{b_{1}, b_{3}, b_{4}, b_{5}\right\}$ be the conditional attribute set, $X=\left\{x_{2}, x_{5}, x_{6}\right\}$ be the decision set. Changing $f\left(x_{8}, b_{4}\right)=2$ to $f^{*}\left(x_{8}, b_{4}\right)=1$.

\begin{tabular}{cccccc}
\multicolumn{6}{c}{ Table 10.} \\
\hline & $b_{1}$ & $b_{3}$ & $b_{4}$ & $b_{5}$ & $d$ \\
\hline$x_{1}$ & 2 & 1 & 1 & 3 & $U-X$ \\
$x_{2}$ & 2 & 1 & 2 & 2 & $X$ \\
$x_{5}$ & 1 & 1 & 2 & 3 & $X$ \\
$x_{6}$ & 1 & 2 & 2 & 2 & $U-X$ \\
$x_{7}$ & 1 & 2 & 2 & 1 & $U-X$ \\
$x_{8}$ & 2 & 1 & 1 & 2 & $U-X$ \\
\hline
\end{tabular}

Using the result of Example 10.

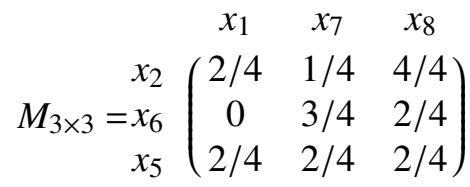

$\underline{G R}_{B}(X)\left(x_{2}\right)=0, Y_{x_{2}}^{\wedge}=\left\{x_{8}\right\} ; \quad \underline{G R}_{B}(X)\left(x_{6}\right)=\frac{1}{4}$, $Y_{x_{6}}^{\wedge}=\left\{x_{7}\right\} ; \quad \underline{G R}_{B}(X)\left(x_{5}\right)=\frac{2}{4}, Y_{x_{5}}^{\wedge}=\left\{x_{1}, x_{7}, x_{8}\right\} ;$ $\underline{G R}_{B}(X)\left(x_{i}\right)=0, x_{i} \in\left\{x_{1}, x_{7}, x_{8}\right\}$.

$\overline{G R}_{B}(X)\left(x_{1}\right)=\frac{2}{4}, Y_{x_{1}}^{\vee}=\left\{x_{2}, x_{5}\right\} ; \overline{G R}_{B}(X)\left(x_{7}\right)=$ $\frac{3}{4}, \quad Y_{x_{7}}^{\vee}=\left\{x_{6}\right\} ; \quad \overline{G R}_{B}(X)\left(x_{8}\right)=\frac{4}{4}, \quad Y_{x_{8}}^{\vee}=\left\{x_{2}\right\} ;$ $\overline{G R}_{B}(X)\left(x_{j}\right)=1, x_{j} \in\left\{x_{2}, x_{5}, x_{6}\right\}$.

From the Step 2 of Algorithm 5.4 we have a new relation matrix $M_{3 \times 3}^{*}$ :

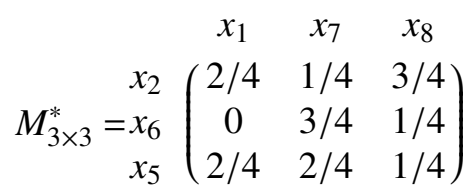


The lower and upper approximations of $X=$ $\left\{x_{2}, x_{5}, x_{6}\right\}$ by changing $f\left(x_{8}, b_{4}\right)$ to $f^{*}\left(x_{8}, b_{4}\right)$ are updated as follows.

From the step 3 of algorithm 5.4 we have the lower approximations:

$\overline{G R}_{B}^{*}(X)\left(x_{2}\right)$ : Since $\underline{G R}_{B}(X)\left(x_{2}\right)=G R_{B}\left(x_{2}, x_{8}\right)$, then $\underline{G R}_{B}^{*}(X)\left(x_{2}\right)=\underset{y \in\left\{x_{1}, x_{7}, x_{8}\right\}}{\wedge}\left(1-G R_{B}^{*}\left(x_{2}, y\right)\right)=\frac{3}{4}$ and $Y_{x_{2}}^{\wedge *}=\left\{y ; \underset{y \in\left\{x_{1}, x_{7}, x_{8}\right\}}{\vee} G R_{B}^{*}\left(x_{2}, y\right)\right\}=\left\{x_{8}\right\} ;$

$\overline{G R}_{B}^{*}(X)\left(x_{6}\right):$ Since $\exists x_{7} \in Y_{x_{6}}^{\wedge}$, such that $\underline{G R}_{B}(X)\left(x_{6}\right)=G R_{B}\left(x_{6}, x_{7}\right)$, and $\underline{G R}_{B}(X)\left(x_{6}\right)>$ $\overline{G R}_{B}^{*}\left(x_{6}, x_{8}\right)$ then $\underline{G R}_{B}^{*}(X)\left(x_{6}\right)=\underline{G R}_{B}(X)\left(x_{6}\right)=\frac{1}{4}$ and $Y_{x_{6}}^{\wedge *}=Y_{x_{6}}^{\wedge}-\left\{x_{8}\right\}=\left\{x_{7}\right\}$;

$\overline{G R}_{B}^{*}(X)\left(x_{5}\right):$ Since $\exists x_{7} \in Y_{x_{5}}^{\wedge}$, such that $\underline{G R}_{B}(X)\left(x_{5}\right)=G R_{B}\left(x_{5}, x_{7}\right)$, and $\underline{G R} R_{B}(X)\left(x_{5}\right)>$ $\overline{G R}_{B}^{*}\left(x_{5}, x_{8}\right)$ then $\underline{G R}_{B}^{*}(X)\left(x_{5}\right)=\underline{G R}_{B}(X)\left(x_{5}\right)=\frac{2}{4}$ and $Y_{x_{5}}^{\wedge *}=Y_{x_{5}}^{\wedge}-\left\{x_{8}\right\}=\left\{x_{1}, x_{7}\right\}$.

From the step 4 of algorithm 5.4 we have the upper approximations:

$$
\begin{aligned}
& \overline{G R}_{B}^{*}(X)\left(x_{j}\right)=\overline{G R}_{B}(X)\left(x_{j}\right), \quad Y_{x_{j}}^{\vee *}=Y_{x_{j}}^{\vee}, \quad x_{j} \in \\
& \left\{x_{1}, x_{7}\right\}^{*} \\
& \overline{G R}_{B}^{*}(X)\left(x_{8}\right)=\underset{y \in\left\{x_{2}, x_{5}, x_{6}\right\}}{\vee} G R_{B}^{*}\left(x_{8}, y\right)=\frac{2}{4} \text { and } Y_{x_{8}}^{\wedge *}= \\
& \left\{y ; \underset{y \in\left\{x_{2}, x_{5}, x_{6}\right\}}{\vee} G R_{B}^{*}\left(x_{8}, y\right)\right\}=\left\{x_{1}, x_{7}\right\} .
\end{aligned}
$$

\section{Algorithm analysis}

The time complexities of all the incrementally updating algorithms proposed in this paper are list in the Table 11 as follow.

From the Table 11 we can see that the proposed incrementally updating algorithms has lower time complexity for every operation on data. In addition to the time cost, it is obviously that every incrementally updating algorithm will use less memory space to get the information updated, because there is no need to rebuild the whole relation matrix in an incrementally updating algorithm.

The incrementally updating algorithms could be seen as a group of functions to update the existing results to keep the relation matrix, approximation and the set of $Y_{x_{j}}^{\vee}$ and $Y_{x_{i}}^{\wedge}$ changing in right way when the information system changed. And we can use different types of the incrementally updating algorithms in any order and with any number of times to adapt to any possible changing in the information system. For example, we can use Algorithm 3.2 once and then use Algorithm 4.1 or 4.2 twice when the basic data need a changing of adding two objects and removing an attribute. And we should note that putting the removing type Algorithm before others will also make the time cost reduced a bit more.

\section{Experimental evaluation}

In this section, we compare the computational time of the non-incremental algorithm and the proposed incremental algorithms on different data sets shown in Table 12. The three data sets used herein came from the University of California, Irvine (UCI) Machine Learning Repository (www.ics.uci.edu/). In data set Dermatology, we delete some attributes with missing values. Experiments were performed on a 2.40GHz Pentium Server with 8GB of memory, running Windows 10. Algorithms were code in Matlab r2012a.

From the following experimental results, it is clear that the incremental algorithm outperform the traditional non-incremental algorithm in different cases, Table 13 ( Where NIA is the nonincrementally updating algorithm and IA is the incrementally updating algorithm ) shows that the proposed methods can effectively update approximations when the information system changes.

\section{Conclusion}

This paper discusses the approaches of incrementally updating of the rough approximations based on the grade indiscernibility relation when complete information system is varied. Taking full use of the existing information make the time cost of the approaches reduced a lot especially when dealing with the large-scale data. In the future, we will discuss the methods of dynamic updating of the rough approximations based on the grade indiscernibility relation in incomplete information system. Moreover, the incremental updating approaches of the decision rules with respect to grade indiscernibility relation is another important and interesting issue to be addressed. 
Table 11.

\begin{tabular}{|c|c|c|c|c|}
\hline Operation & $\begin{array}{l}\text { Non-incremental } \\
\text { algorithm }\end{array}$ & $\begin{array}{c}\text { Time } \\
\text { Complexity }\end{array}$ & $\begin{array}{l}\text { Incremental } \\
\text { algorithm }\end{array}$ & $\begin{array}{c}\text { Time } \\
\text { Complexity }\end{array}$ \\
\hline Adding an attribute $b$ & \multirow{10}{*}{ Algorithm 2.1} & \multirow{10}{*}{$O(|X||U-X||B|)$} & Algorithm 3.1 & $O(|X||U-X|)$ \\
\hline Removing an attribute $b$ & & & Algorithm 3.2 & $O(|X||U-X|)$ \\
\hline Adding an object $x \in U-X$ & & & Algorithm 4.1 & $O(|X||B|)$ \\
\hline Adding an object $x \in X$ & & & Algorithm 4.2 & $O(|U-X||B|)$ \\
\hline Removing an object $x \in U-X$ & & & Algorithm 4.3 & $O(|X|)$ \\
\hline Removing an object $x \in X$ & & & Algorithm 4.4 & $O(|U-X|)$ \\
\hline$x \in U-X \rightarrow x \in X$ & & & Algorithm 5.1 & $O(|U-X||B|)$ \\
\hline$x \in X \rightarrow x \in U-X$ & & & Algorithm 5.2 & $O(|X||B|)$ \\
\hline$f(x, b) \rightarrow f^{*}(x, b), x \in X$ & & & Algorithm 5.3 & $O(|U-X||B|)$ \\
\hline$f(x, b) \rightarrow f^{*}(x, b), x \in U-X$ & & & Algorithm 5.4 & $O(|X||B|)$ \\
\hline
\end{tabular}

Table 12.

\begin{tabular}{cccccc}
\hline ID & Data set & Number of objects & Number of attributes & Decision classes & Missing value \\
\hline 1 & Zoo & 101 & 17 & 7 & No \\
2 & Dermatology & 366 & 33 & 7 & Yes \\
3 & Chess & 3196 & 36 & 2 & No \\
\hline
\end{tabular}

Table 13. Comparisons of computational time between nonincremental algorithm and incremental simplified algorithm when objects and attributes increase simultaneously(s).

\begin{tabular}{|c|c|c|c|c|c|c|}
\hline \multirow{2}{*}{ Operation } & \multicolumn{2}{|c|}{ Zoo } & \multicolumn{2}{c|}{ Dermatology } & \multicolumn{2}{c|}{ Chess } \\
\cline { 2 - 7 } & NIA & IA & NIA & IA & NIA & IA \\
\hline Adding an attribute $b$ & 0.0115 & 0.0083 & 0.1735 & 0.0064 & 15.8108 & 0.2609 \\
\hline Removing an attribute $b$ & 0.0115 & 0.0077 & 0.1774 & 0.0065 & 15.8190 & 0.2316 \\
\hline Adding an object $x \in U-X$ & 0.0123 & 0.0030 & 0.1582 & 0.0052 & 15.9404 & 0.1340 \\
\hline Adding an object $x \in X$ & 0.0120 & 0.0081 & 0.1485 & 0.0074 & 16.2771 & 0.1263 \\
\hline Removing an object $x \in U-X$ & 0.0101 & 0.0044 & 0.1708 & 0.0049 & 15.9115 & 0.1308 \\
\hline Removing an object $x \in X$ & 0.0114 & 0.0067 & 0.1697 & 0.0057 & 16.0953 & 0.1302 \\
\hline$x \in U-X \rightarrow x \in X$ & 0.0117 & 0.0055 & 0.1726 & 0.0054 & 15.9011 & 0.1641 \\
\hline$x \in X \rightarrow x \in U-X$ & 0.0112 & 0.0099 & 0.1744 & 0.0067 & 15.7100 & 0.1548 \\
\hline$f(x, b) \rightarrow f^{*}(x, b), x \in X$ & 0.0120 & 0.0079 & 0.1795 & 0.0067 & 15.9708 & 0.1184 \\
\hline$f(x, b) \rightarrow f^{*}(x, b), x \in U-X$ & 0.0115 & 0.0030 & 0.1623 & 0.0060 & 15.8709 & 0.1442 \\
\hline
\end{tabular}




\section{Acknowledgements}

This work was partially supported by the National Natural Science Foundation of China (Grant No. 61473239, 61175044, 61372187), the Fundamental Research Funds for the Central Universities of China (Grant No. 2682014ZT28), and the open research fund of key laboratory of intelligent network information processing, Xihua University (szjj2014052).

\section{References}

1. Z. Pawlak, Rough sets, International Journal of Computer and Information Sciences 11(1982)341-356.

2. Z. Pawlak, Rough sets-theoretical aspects of reasoning about data, Kluwer Academic Publishers (1991).

3. Z. Pawlak, Rough set theory and its applications to data analysis, Cybernetics and Systems 29(1998)661688.

4. A. Skowron, Extracting laws from decision tables: a rough set approach, Computational Intelligence 11(1995)371-388.

5. Y.Y. Yao, P.J. Lingras, Interpretations of belief functions in the theory of rough sets, Information Sciences 104(1998)81-106.

6. W.X. Zhang, J.S. Mi, W.Z. Wu, Approaches to knowledge reductions in inconsistent systems, International journal of intelligent systems 18(2003)989-1000.

7. Z. Bonikowski, E. Bryniarski, U. Wybraniec, Extensions and intentions in the rough set theory, Information Sciences 107(1998)149-167.

8. D. Dubois, H. Prade, Rough fuzzy set and fuzzy rough sets, International Journal of General System 17(1990)191-209.

9. W. Ziarko, Variable precision rough set model, Journal of Computer and System Sciences 46(1993)39-59.

10. Y.Y. Yao, Relational interpretations of neighborhood operators and rough set approximation operators, Information Sciences 111(1998)239-259.

11. Y.Y. Yao, Probabilistic rough set approximations, International Journal of Approximate Reasoning 49(2008)255-271.

12. R.S. Michalski, Knowledge repair mechanisms: evolution vs. revolution, Proceedings of the Third International Machine Learning Workshop(1985)116-119.

13. A. Bouchachia, R. Mittermeir, Towards incremental fuzzy classifiers, Soft Computing 11(2007)193-207.

14. Y. Cheng, The incremental method for fast computing the rough fuzzy approximations, Data and Knowledge Engineering 70(2011)84-100.
15. C.C. Chan, A rough set approach to attribute generalization in data mining, Information Sciences 107(1998)169-176.

16. T.R. Li, D. Ruan, W. Geert, J. Song, Y. Xu, A rough set based characteristic relation approach for dynamic attribute generalization in data mining, KnowledgeBased Systems 20(2007)485-494.

17. J.B. Zhang, T.R. Li, D. Ruan, and D. Liu, Neighborhood rough sets for dynamic data mining, International Journal of Intelligent Systems 27(2012)317342.

18. Y. Zhao, Y.Y. Yao, Data analysis based on discernibility and indiscernibility, Information Sciences 177(2007)4959-4976.

19. K.Y. Qin, J.F. Luo, Rough set model based on grade indiscernibility relation, Computer Science 42(2015)240-243.

20. J.F. Luo, K.Y. Qin, The rough set model based on grade indiscernibility relation, 2015 IEEE International Conferences on Computer and Information Technology (2015)1366-1371.

21. K.Y. Qin, J.F. Luo, Z. Pei, Rough Approximations Based on Valued Tolerance Relations, Fundamenta Informaticae 142(2015)183-194.

22. M. Lichman, UCI Machine Learning Repository, http://archive.ics.uci.edu/ml, University of California, Irvine, School of Information and Computer Sciences 2013.

23. N. Shan and W. Ziarko, Data-based acquisition and incremental modification of classification rules, Computational Intelligence 11(1995)357-370.

24. Y.Y. Yao, T.Y. Lin, Generalization of rough sets using modal logics, Intelligent Automation and Soft Computing 2(1996)103-119.

25. Q.S. Zhou, T.R. Li, et al, Research on properties of approximations in rough sets based on characteristic relation when object varies with time, Computer Science 39(2012)191-197.

26. S.Y. Li, T.R. Li, D. Liu, Incremental updating approximations in dominance-based rough sets approach under the variation of the attribute set, Knowledge-Based Systems 40(2013)17-26.

27. H.M. Chen, T.R. Li, C. Luo, S.J. Horng, G.Y. Wang, A decision-theoretic rough set approach for dynamic data mining, IEEE Transactions on Fuzzy Systems 23(2015)1958-1970.

28. H.M. Chen, T.R. Li, D. Ruan, J.H. Lin, C.X. Hu, A rough-set-based incremental approach for updating approximations under dynamic maintenance environments, IEEE Transactions on Knowledge and Data Engineering 25(2013)274-284.

29. L. Feng, T.R. Li, D. Ruan, S. Gou, A vague-rough set approach for uncertain knowledge acquisition, Knowledge-Based Systems 24(2011)837-843. 